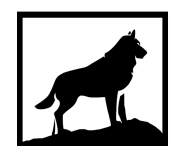

Michigan Technological

1 8 8 5 University
Michigan Technological University

Digital Commons @ Michigan Tech

\title{
APPLICATION OF SHORT TANDEM TARGET MIMIC (STTM) TECHNIQUE FOR FUNCTIONAL ANALYSIS OF MICRO-RNA396 IN TRANSGENIC POPLAR TREES
}

Surattana Boonsai

Michigan Technological University, sboonsai@mtu.edu

Copyright 2019 Surattana Boonsai

\section{Recommended Citation}

Boonsai, Surattana, "APPLICATION OF SHORT TANDEM TARGET MIMIC (STTM) TECHNIQUE FOR FUNCTIONAL ANALYSIS OF MICRO-RNA396 IN TRANSGENIC POPLAR TREES", Open Access Master's Thesis, Michigan Technological University, 2019.

https://doi.org/10.37099/mtu.dc.etdr/782

Follow this and additional works at: https://digitalcommons.mtu.edu/etdr

Part of the Agricultural Education Commons, Biotechnology Commons, Botany Commons, Forest Biology Commons, Genetics Commons, Other Genetics and Genomics Commons, Plant Biology Commons, and the Plant Breeding and Genetics Commons 


\title{
APPLICATION OF SHORT TANDEM TARGET MIMIC (STTM) TECHNIQUE FOR FUNCTIONAL ANALYSIS OF MICRO-RNA396 \\ IN TRANSGENIC POPLAR TREES
}

\section{By}

Surattana Boonsai

\begin{abstract}
A THESIS
Submitted in partial fulfillment of the requirements for the degree of MASTER OF SCIENCE In Biological Sciences
\end{abstract}

\section{MICHIGAN TECHNOLOGICAL UNIVERSITY \\ 2019}

(C) 2019 Surattana Boonsai 
This thesis has been approved in partial fulfillment of the requirements for the Degree of MASTER OF SCIENCE in Biological Sciences.

\author{
Department of Biological Sciences
}

\author{
Thesis Co-Advisor: $\quad$ Dr. Chandrashekhar P. Joshi \\ Thesis Co-Advisor: Dr. Guiliang Tang \\ Committee Member: Dr. Rupali Datta \\ Department Chair: Dr. Chandrashekhar P. Joshi
}




\section{Table of Contents}

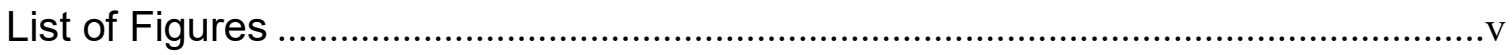

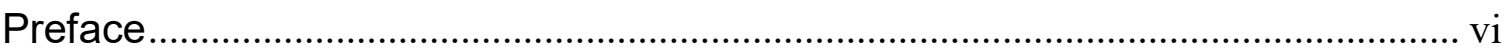

Acknowledgements ............................................................................................... vii

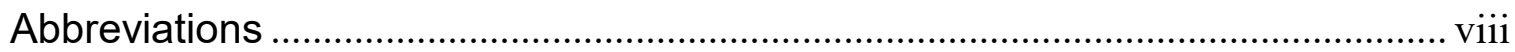

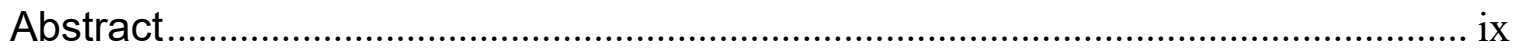

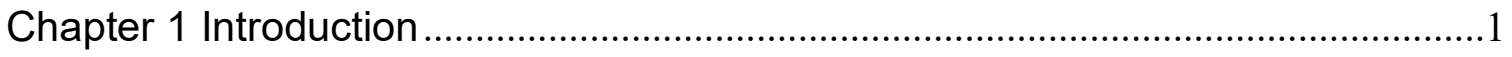

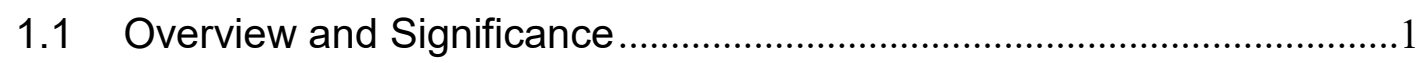

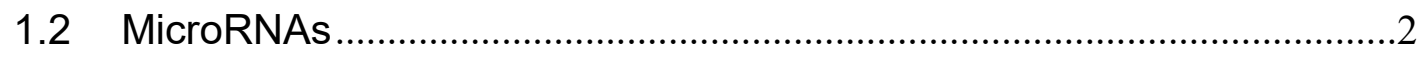

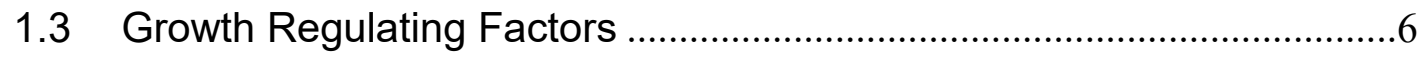

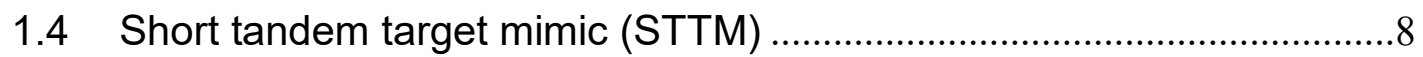

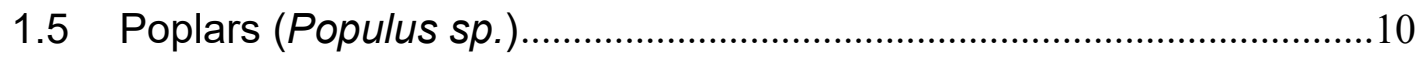

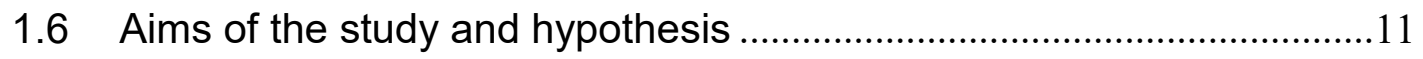

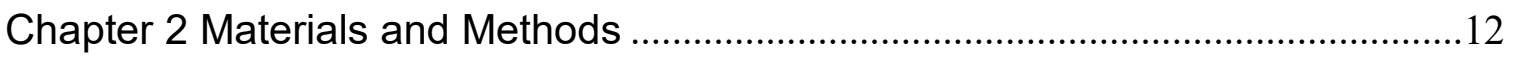

2.1 Plasmid construction, plant transformation and growth condition ............12

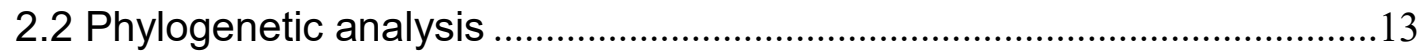

2.3 Total RNA isolation and stem-loop RT-qPCR ...........................................13

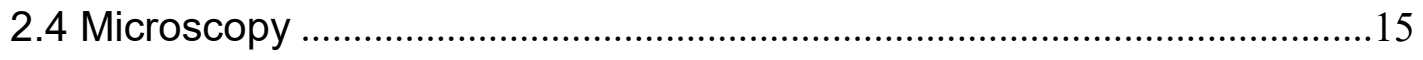

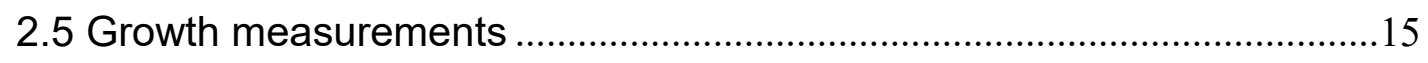

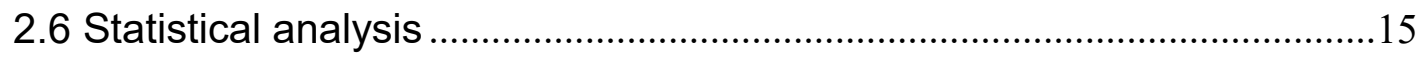

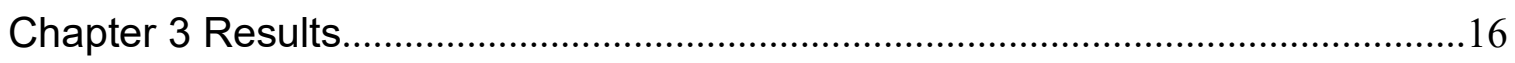

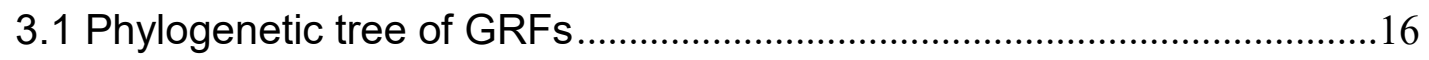

3.2 MiR396 was effectively knocked down in poplar by STTM ......................17

3.3 Blockage of miR396 induced taller plants with larger leaves ....................18

3.4 STTM396 plants have altered leaf cell size and cell number...................22

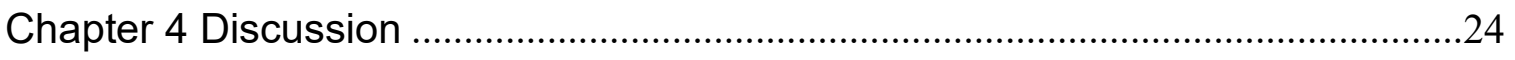

4.1 MiR396 was effectively knocked down in poplar by STTM ......................24

4.2 Blockage of miR396 induced taller plants with larger leaves ....................24 
4.3 STTM396 plants have altered leaf cell size and cell number.

4.4 Expression level of GRFs changed in STTM396 plants ...........................25

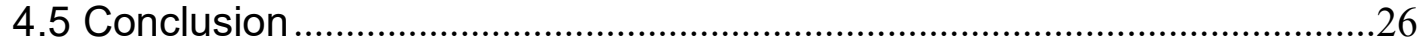

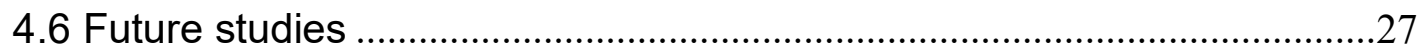

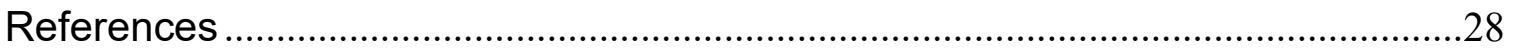




\section{List of Figures}

Figure 1.1: Diagram of plant miRNAs biogenesis and function (Adapted from $\mathrm{Ma}$ et al., 2009; Axtell et al., 2011).

Figure 1.2: Alignment of miR396s in Arabidopsis, poplar, rice, maize, and tomato.

Figure 1.3: MiR396 group $A$ and $B$ show one nucleotide difference at the end of 3' (G vs U).

Figure 1.4: Ptc-GRFs genes distribution in poplar genome..................................

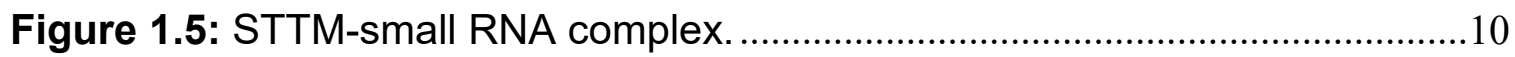

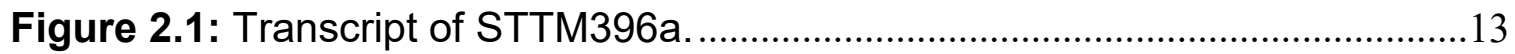

Figure 3.1: Phylogenetic tree of AthGRFs and PtcGRFs....................................16

Figure 3.2: MiR396 was effectively knocked down in poplar by STTM.................18

Figure 3.3: Length of the first leaf from the bottom in centimeters $(\mathrm{cm})$..............19

Figure 3.4: Length of the second leaf from the bottom in centimeter $(\mathrm{cm})$..........19

Figure 3.5: Estimated height in centimeter $(\mathrm{cm})$ of wild type and STTM396 plants

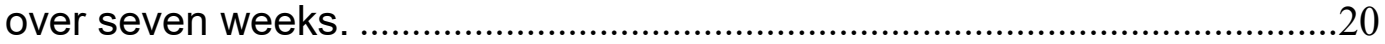

Figure 3.6: Comparison plant stature (A) and leaf length (B) between wild type

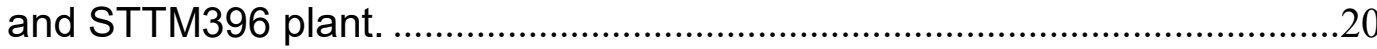

Figure 3.7: Estimated stem girth in millimeter $(\mathrm{mm})$ of wild type and STTM396 plants over seven weeks.

Figure 3.8: Leaf count (n) of wild type and STTM396 plants over seven weeks. 21

Figure 3.9: Transverse section of wild type and STTM396 leaves, particularly at mid vein region.

Figure 3.10: STTM396 resulted in smaller cell number in STTM leaves..............22

Figure 3.11: STTM396 resulted in larger cell size in STTM leaves. ......................23

Figure 4.1: Proposed schematic relations between miR396 and GRFs affecting growth and development in poplar. 


\section{Preface}

In this study, the experiments were designed together by

Dr.Chandrashekhar Joshi, Dr. Guilliang Tang and myself.

The PCAMBIA1300STTM396a construct was made by Dr. Haiping Liu.

All transgenic plants were generated and analyzed by the author of this thesis. 


\section{Acknowledgements}

First, I would like to thank my advisor, Dr. Chandrasekhar Joshi for all the advice and support for almost three years during my MS studies at Michigan Tech. I also would like to thank my thesis co-advisor, Dr. Guiliang Tang for his advice during experimental design and my thesis committee member Dr. Rupali Datta for her valuable help during my research work and thesis writing. I also would like to thank Biological Sciences staff and faculty for their help during my research. I wish to thank the Royal Thai Government and the Biological Sciences department for the financial support.

Second, I would like to thank my friends for their help and support. I would like to thank Dr.Joshi's lab members, especially Dr. Kavitha Kumar who guided and taught me in several molecular biology and plant physiology techniques and helped me in trouble shooting in many experiments. I also appreciate help from my friends in Biological Sciences department, especially Taija Hakha who has been motivating and supporting me in study, research, and other life aspects. I also thank Tanner Keyzers, and Ryan Ghannam for their helps on guiding and proof reading my thesis writing.

Last but not least, I would like to thank my family for always supporting me all the way. Also, my Thai friends at Michigan Tech are also my family for supporting me and helping me during my Michigan Tech life. 


\section{Abbreviations}

Ath-GRF Arabidopsis thaliana-Growth regulating factor

Bra-GRF Brassica rapa subsp. Pekinensis- growth regulating factor

GRF growth regulating factor

MiR microRNA

Osa-GRF Oryza sativa-Growth regulating factor

Ptr-GRF Populus trichocarpa-Growth regulating factor

STTM short target tandem mimic

Sly-GRF Solanum lycopersicum-Growth regulating factor

WT wild type

Zma-GRF Zea mays- Growth regulating factor 


\section{Abstract}

Short Tandem Target Mimic (STTM) is a commonly used technique for functional studies of a number of genes in several plant model systems. However, very little is known about application of STTM technique in tree species. In this study, STTM was applied to knock down microRNA396 (miR396) in transgenic poplar trees for the first time. STTM396 expression resulted in dramatic decrease in miR396 expression levels leading to taller plants with larger leaves and larger leaf cell size. Additionally, an expression analysis of growth regulating factor genes (GRFs) that are members of miR396 target gene family showed up-regulation of GRF07 gene suggesting that miR396 might be negatively regulating growth and cell expansion in leaves via GRF07 suppression. On the contrary, down-regulation of GRF06 and GRF18 was observed in the same transgenic trees suggesting the complexity of gene regulatory relationship between expression of miR396 and GRFs in poplar. Remarkably, GRF10 and GRF17 were barely expressed in leaves tissues suggesting they might be expressed in other tissues in a tissue-specific manner. Overall, these results suggest that the STTM is an effective approach for functional analyses of miRNAs in poplars, a model tree species. In addition, miR396 may be playing some important roles in growth and cell expansion of leaves via regulating expression of GRF genes in poplars. 


\section{Chapter 1 Introduction}

\subsection{Overview and Significance}

MicroRNAs (miRNAs) are short, non-coding RNAs consisting of 20-24 nucleotides ( $\mathrm{nt}$ ) that can silence gene expression of target genes via messenger RNA (mRNA) degradation and/or mRNA translational repression (Pareek et al., 2015). In plants, miRNAs have been reported to play important roles in various biological processes including in growth and development (Li and Zhang, 2016).

For example, miR396 is one of the most conserved miRNAs among plant species (Sun, 2012). Computational prediction suggests that miR396 targets expression of growth regulating factors (GRFs), which are plant-specific transcriptional factors (https://plantgrn.noble.org/psRNATarget/analysis). Because miR396 as well as GRFs are highly conserved among plants, miR396 are known to regulate cell growth and proliferation via regulating expression of GRF genes in various plants such as Arabidopsis, rice, tomato, maize, and Chinese cabbage but little is known about miR396 and GRFs in tree species (Liang et al., 2014; Gao et al., 2015; Cao et al., 2016; Zhang et al., 2018; Wang et al., 2014).

Poplar is an excellent tree model system for forest genetics and woody plant research due to its rapid growth rate, well-studied small genome and capability to be transformed using Agrobacterium-mediated gene expression vectors (Tuskan et al., 2006). In order to study miRNAs functions, Short Tandem Target Mimic (STTM) is a relatively new technique to block functions of miRNAs (Tang et al., 2012). STTM has two mimic miRNA binding sites, which partially complement with target miRNA. STTM has been used to block miRNAs effectively in animals and several plant species (Tang et al., 2012) but has been applied to study miRNAs in trees only a few times (Su et al., 2018; Kumar, 2019). Therefore, applying STTM to study miR396 and GRFs in poplar will elucidate functions of miR396 and GRF gene family and examine the efficiency of STTM in poplar trees. 


\subsection{MicroRNAs}

MicroRNAs (miRNAs) are 20-24 nt short endogenous non-coding small RNAs that can inactivate target mRNA and silence gene expression (Reinhart et al., 2002). Briefly, primary miRNA (Pri-miRNA) is encoded from a miRNA gene that forms a hairpin loop structure in the nucleus. Then, Pri-miRNA is modified through several steps by Drosha-DGCR8 and exported to cytoplasm by HASTY, a homolog of an Exportin 5 (Exp5) in animals. Afterward, it is incorporated into Dicer and Argonaute proteins to form RNA-induced silencing complex (RISC) and bind to target mRNA. In plants, the RISC can either degrade target mRNA directly (mRNA degradation) if it can match with the target mRNA perfectly or it can suppress translation of the target mRNA (inhibition of translation) in case of a partial complement (Bartel, 2004; Axtell et al, 2011) as shown in Figure 1.1.

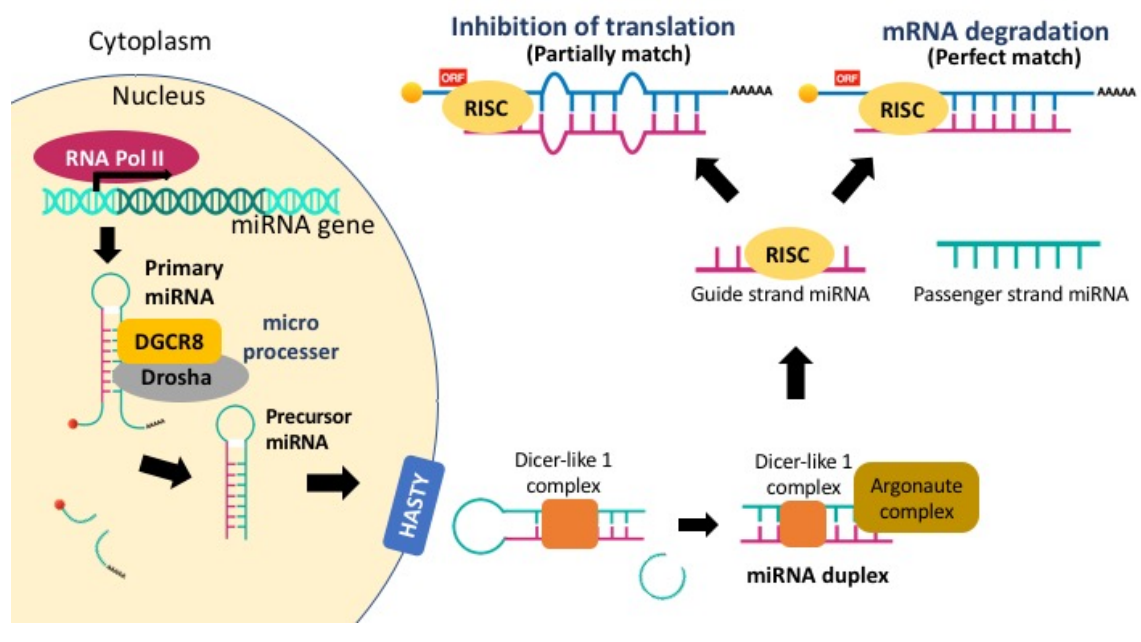

Figure 1.1: Diagram of plant miRNAs biogenesis and function (Adapted from Ma et al., 2009; Axtell et al., 2011).

Plant miRNAs play important roles in growth and development ( $\mathrm{Li}$ and Zhang, 2016). For example, in Arabidopsis, miR160 controls root growth and gravitropism by negatively regulating auxin response factor (ARF) 10, ARF16 and ARF17 (Khan et al., 2011). The miR390 controls growth and senescence through ARF2 and ARF3 expression regulation (Mecchia et al., 2013). In addition, miRNAs 
are involved with hormone signaling pathways. For example, miR165/166 regulate auxin (IAA) content and sensitivity by enhanced expression of auxin biosynthesis genes in Arabidopsis (Jia et al., 2015). Several miRNAs are important in biotic and abiotic stress responses (Khraiwesh et al., 2012). MiR397 positively regulates cold tolerance via C-repeat binding factor (CBF) dependent pathway (Dong and Pei, 2014). Also, miR402 regulates seed germination timing and cold tolerance (Kim et al., 2010).

MicroRNA396 (miR396) is an evolutionarily conserved miRNA among monocot and dicot plants. So far miR396 has 60 members of miR396 from 21 plant species are known (Sun, 2012). According to microRNA database (http://www.mirbase.org), miR396 has two mature types in Arabidopsis and tomato, while seven members in poplar, nine members in rice, and eight members in maize (Griffiths-Jones et al., 2006). Alignment of miR396s in Arabidopsis, maize, rice, tomato, and poplar show two miR396 groups that have identical mature sequence (Figure 1.2). Remarkably, both mature types of miR396 have only one nucleotide difference at their 3' end (G vs U) (Figure 1.3). 


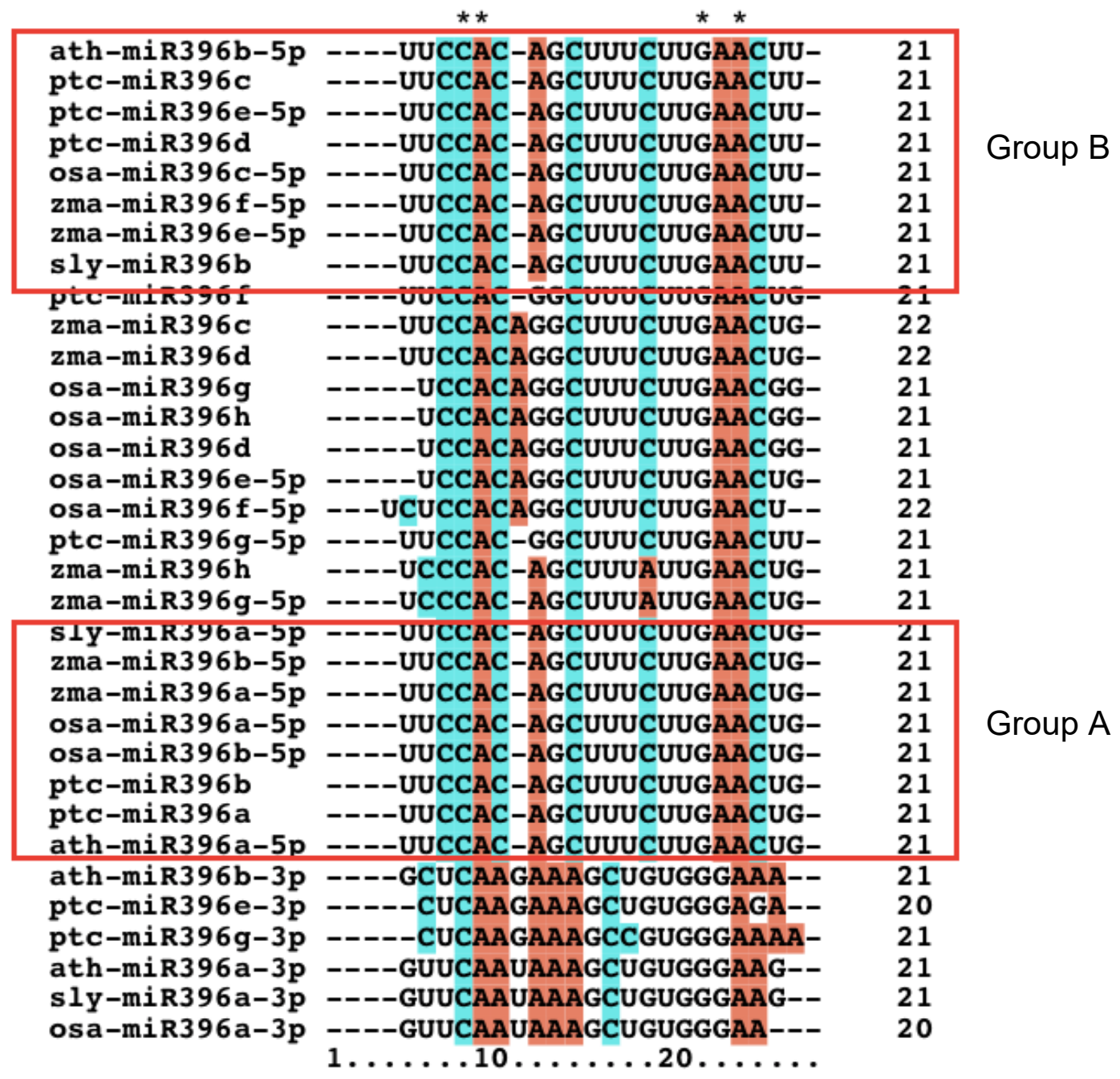

Figure 1.2: Alignment of miR396s in Arabidopsis, poplar, rice, maize, and tomato.

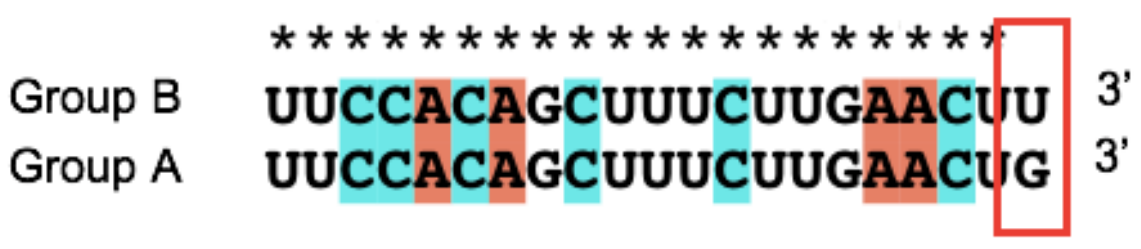

Figure 1.3: MiR396 group $A$ and $B$ show one nucleotide difference at the end of 3' ( $G$ vs $U$ ). 
Tomato (Solanum lycopersicum) has two mature miR396 types including miR396a and miR396b, which have only one nucleotide difference at the end of 3' ( $G$ vs $U$ ) but miR396a show dramatically higher expression than miR396b in cotyledon, flower, sepal, and fruit suggesting that miR396a might play more important roles in tomato growth and development than miR396b. Furthermore, STTM396a/396a-88 construct could knock down both miR396a and miR396b in tomato (Cao et al., 2016). In Arabidopsis, there are two miR396 genes in miR396 family including miR396a and miR396b encoding two mature miRNAs, which are different in the last nucleotide ( $G$ in miR396a and $U$ in miR396b). In Populus trichocarpa, miR396 family has seven genes (miR396a-g). Mature sequence of ptc-mir396a and ptc-miR396b are identical to ath-miR396a whereas mature sequence of ptc-miR396c is identical to ath-miR396b. In addition, mature sequence of ptc-miR396f and ptc-miR396g have one and two nucleotides variation compared to ath-miR396a respectively (Figure 1.2).

MiR396 has been studied extensively in Arabidopsis, rice and some other crop plants such as tomato and maize. For example, miR396 targets Arabidopsis thaliana-growth regulating factors (ath-GRFs) gene family, which are transcription factors for cell proliferation in leaves (Rodriguez et al., 2010). Also, miR396 negatively regulates size of flower, sepal, fruit and cotyledon through suppressed GRFs expression in tomato (Cao et al., 2016). In addition, Peng et al. observed larger tomato with bigger flowers, leaves, and fruit when miR396 was knocked down (Peng et al., 2018). Besides, over-expression of ptc-miR396c in tobacco resulted in cotyledon fusion, lack of shoot apical meristem, delayed and altered floral organ specification. The results from this heterologous study suggested a role of miR396 in floral organs specification (Baucher et al., 2013). Nevertheless, functions of poplar-miR396a-b remain unexplored. 


\subsection{Growth Regulating Factors}

Growth regulating factors (GRFs) are a small plant-specific transcription factor family. The main common character of GRFs is containing two conserved regions including QLQ and WRC domain, which are in N-terminal part of GRFs. (Omidbakhshfard et al., 2015). The QLQ domain can be found in all eukaryotes, while WRC is a plant-specific domain (Van der Knaap et al., 2000).

According to PlantTFDB database (http://planttfdb.cbi.pku.edu.cn), GRFs family includes 9 proteins in Arabidopsis, 12 proteins in rice and tomato, 14 proteins in maize, and 19 proteins in poplar (Table 1.1). Computational prediction, based on complementarity between miRNA and sequence of the target genes, suggested that 7 out of 9 ath-GRFs, 8 out of 12 osa-GRFs, 8 out of 12 sly-GRFs, 12 out of $14 \mathrm{zma}-G R F s$, and 18 out of 19 ptc-GRFs are targeted by miR396 (psRNATarget) (https://plantgrn.noble.org/psRNATarget/analysis).

GRFs have been studied experimentally in several plant species. For example, knocking down miR396 resulted in up-regulated osa-GRF6 and increased yield in rice through inflorescence development, altered auxin biosynthesis and auxin-response factors, and branch and spikelet development (Gao et al., 2015). In addition, over-expression of osa-GRF1 in Arabidopsis resulted in impaired stem growth suggesting that it plays regulatory roles in GAinduce stem elongation (Van der Knaap et al., 2000). In Arabidopsis, overexpression of miR396 caused abnormal flowers and down-regulation of all athGRFs (ath-GRF1-9) (Liang et al., 2014). In Chinese cabbage (B. rapa L ssp. pekinensis), 15 bra-GRFs out of 17 were expressed mostly in young leaves but fewer bra-GRF genes were expressed in old leaves. Bra-GRF8 showed the largest expression difference between young and old leaves while bra-GRF16 was strongly expressed in roots (Wang et al., 2014). However, the functions of GRFs in poplar remain unclear. 
Table 1.1: Number of Ptc-GRFs genes and transcription factors in poplar.

\begin{tabular}{|c|c|c|c|c|}
\hline Gene name & $\begin{array}{l}\text { Transcription } \\
\text { factor ID }\end{array}$ & Description $^{\mathrm{a}}$ & Chromosome & Length $^{\mathrm{b}}(\mathrm{aa})$ \\
\hline Ptc-GRF01 & Potri.001G082700.1 & GRF 8 & Chr01 & 514 \\
\hline \multirow[b]{2}{*}{ Ptc-GRF02 } & Potri.001G114000.1 & GRF 10 & Chr01 & 203 \\
\hline & Potri.001G114000.2 & GRF 10 & Chr01 & 178 \\
\hline Ptc-GRF03 & Potri.001G132600.1 & GRF 4 & Chr01 & 343 \\
\hline Ptc-GRF04 & Potri.001G169100.1 & GRF 5 & Chr01 & 347 \\
\hline Ptc-GRF05 & Potri.002G115100.1 & - & Chr02 & 468 \\
\hline Ptc-GRF06 & Potri.003G065000.1 & GRF 5 & Chr03 & 343 \\
\hline \multirow{4}{*}{ Ptc-GRF07 } & Potri.003G100800.1 & GRF 3 & Chr03 & 348 \\
\hline & Potri.003G100800.2 & GRF 3 & Chr03 & 337 \\
\hline & Potri.003G100800.3 & GRF 3 & Chr03 & 340 \\
\hline & Potri.003G100800.4 & GRF 3 & Chr03 & 343 \\
\hline Ptc-GRF08* & Potri.003G118100.1 & GRF 10 & Chr03 & 202 \\
\hline Ptc-GRF09 & Potri.006G115200.1 & GRF 4 & Chr06 & 378 \\
\hline Ptc-GRF10 & Potri.006G143200.1 & GRF 1 & Chr06 & 386 \\
\hline Ptc-GRF11 & Potri.007G007100.1 & GRF 1 & Chr07 & 615 \\
\hline \multirow[b]{2}{*}{ Ptc-GRF12 } & Potri.012G022600.1 & GRF 4 & Chr12 & 581 \\
\hline & Potri.012G022600.2 & GRF 4 & Chr12 & 536 \\
\hline Ptc-GRF13 & Potri.013G077500.1 & GRF 4 & Chr13 & 341 \\
\hline Ptc-GRF14 & Potri.014G007200.1 & GRF 1 & Chr14 & 608 \\
\hline Ptc-GRF15 & Potri.014G012800.1 & - & Chr14 & 610 \\
\hline \multirow[t]{3}{*}{ Ptc-GRF16 } & Potri.014G071800.1 & GRF 9 & Chr14 & 459 \\
\hline & Potri.015G006200.1 & GRF 4 & Chr15 & 448 \\
\hline & Potri.015G006200.3 & GRF 4 & Chr15 & 412 \\
\hline Ptc-GRF17 & Potri.015G006200.4 & GRF 4 & Chr15 & 436 \\
\hline Ptc-GRF18 & Potri.018G065400.1 & GRF 1 & Chr18 & 383 \\
\hline Ptc-GRF19 & Potri.019G042300.1 & - & Chr19 & 329 \\
\hline
\end{tabular}

* Potri.003G118100.1 is non-miR396 target.

a Description was obtained from NCBI.

b Length was referred to the translated Pt-GRF proteins.

Data were obtained from http://planttfdb.cbi.pku.edu.cn 


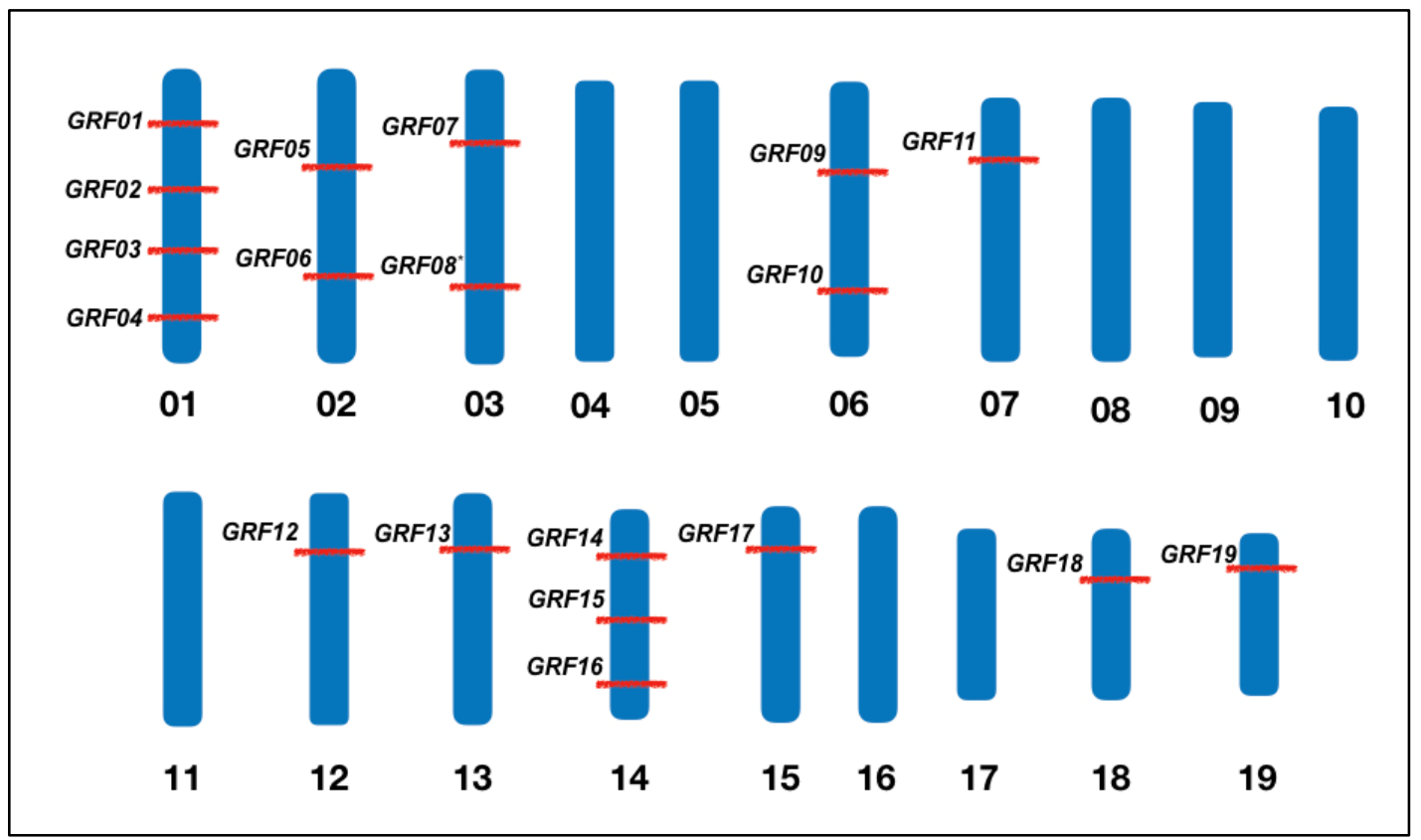

Figure 1.4: Ptc-GRFs genes distribution in poplar genome.

The chromosome number is indicated at the bottom of each chromosome representation.

Ptc-GRFs genes are distributed unevenly in poplar genome as shown in Figure 1.4, which is similar to the previous results in Arabidopsis (Kim et al., 2003), tomato (Choi et al., 2004), and rice (Zhang et al., 2008). In poplar, the chromosome 01 has four ptc-GRFs (01-04) genes, whereas chromosome 14 has three genes (GRF14-16). In addition, two genes each are located on chromosome 03 and 06. Furthermore, one gene each is positioned on chromosome $02,07,12,13,17,18$ and 19.

\subsection{Short tandem target mimic (STTM)}

Since plant miRNAs were found in 2002 by several research groups (Llave et al., 2002; Mette et al., 2002; Park et al., 2002; Reinhart et al. 2002;), some approaches have been used to identify and characterize miRNAs. Functional study of genes traditionally relied on reverse genetics to knock down genes of interest and observe loss of functions or altered phenotypes. However, it was difficult to 
knockdown miRNAs due to their small size and each miRNA has many target genes. After the discovery of target mimic (TM), short tandem target mimic (STTM) was developed to target small RNAs (Tang et al., 2012).

STTM is a synthetic, short, non-coding RNA ( 100 nt). The STTM construct is made as described earlier (Tang et al., 2012) and is expressed by CaMV $35 \mathrm{~S}$ promoter. The STTM transcript consists of a stem-loop linker at the middle and two small RNA binding sites on both sides. The stem-linker may play a role in STTM stability meanwhile the binding sites partially complement with small RNAs, except CUA region, which enhance STTM to trap small RNAs without being cleaved by them (Figure 1.5). Additionally, the 48-88 nt spacer with a weak hair-pin loop structure is required for STTM. STTM can target miRNA functions by degrading miRNAs directly and suppressing mRNA translation (Tang et al., 2012).

STTM has been used to block miRNAs successfully in fungi, animals and several plants. STTM can knockdown several fungal miRNAs in Metarhizium robertsii and Aspergillus flavus and leads to up-regulation of target genes involving development and conidial production (Wang et al., 2019). In addition, miR30-d expression was drastically reduced in STTM30-d mouse cells, demonstrating that STTM also functions efficiently in animal cells (Tang et al., 2012). In Arabidopsis, STTM165/166 reduced miR165/166 expression level and increased HD-ZIP III transcription factors genes expression, their target genes, leading to a series of developmental changes (Yan et al., 2012). In addition, knocking down miR160 in tomato using STTM160 showed decrease in miR160 expression levels and upregulation of SIARF10A, SIARF10B and SIARF17, its target genes. Also, STTM160 tomato plants displayed changes in ovary patterning, floral organ abscission and lateral organ outgrowth (Damodharan et al., 2016). Nonetheless, STTM approach has recently been applied to study miRNAs in trees only a few times before (Su et al., 2018; Kumar, 2019). 


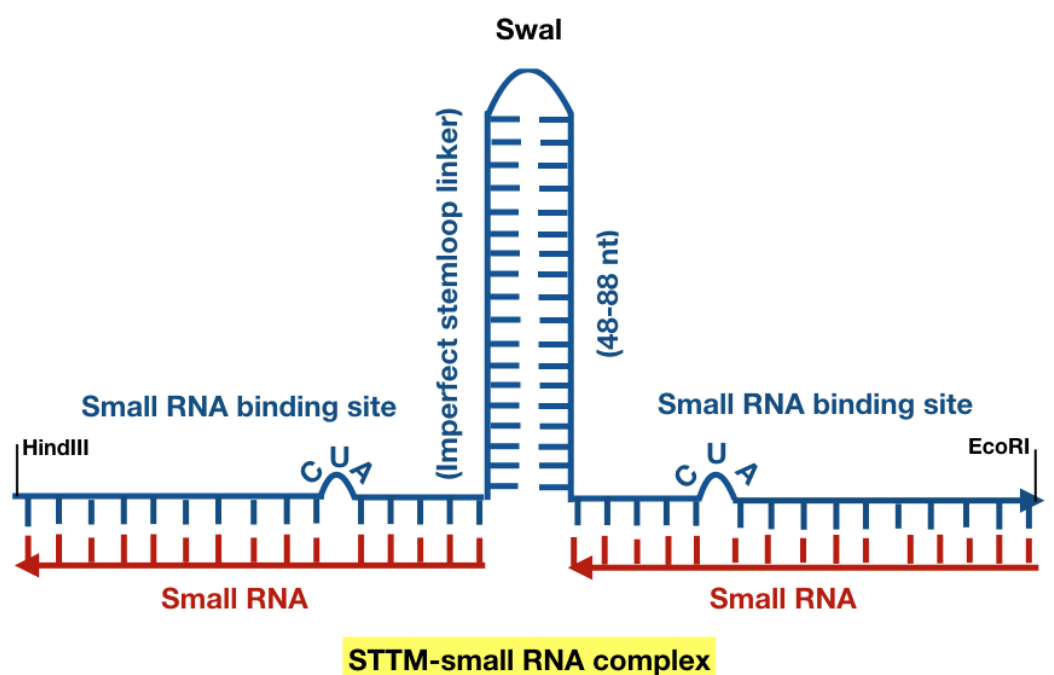

Figure 1.5: STTM-small RNA complex.

The STTM transcript binds with small RNA to form the STTM-small RNA complex through small RNA binding sites (Adapted from Tang et al., 2012.).

\subsection{Poplars (Populus sp.)}

Several characteristics of trees, especially having a large size and long life span, differentiate them from other model plants and make molecular and cellular levels study challenging. Populus is a good model tree genus suitable for forest genetics due to its modest genome, rapid growth, and compatibility with DNA transformation by Agrobacterium tumefaciens. Populus includes $\sim 30$ species including aspens, cottonwoods, and poplars. Populus trichocarpa or black cotton wood was selected as the tree model species for genome sequencing in 2006. Genome sequencing uncovered that it has 19 chromosomes with compact 500 Mbp-big genomes. There is a total of 169 identified miRNA genes representing 21 families (Tuskan et al., 2006). 


\subsection{Aims of the study and hypothesis}

Overall, miR396 has been reported to play roles in growth and development of plant species via regulation of GRFs target gene expression. Since ptc-miR396a has never been studied in any tree species and STTM has been applied to study miRNAs in tree species only a few times, in this study, we applied STTM to study functions of miR396a in poplar. Aims of this study include to investigate efficiency of STTM in poplars and to study functions of ptc-miR396a especially in growth and development through expression of GRF genes. We hypothesized that STTM396a will knock down miR396a expression in transgenic poplar trees and will show decreased miR396a expression level. Also, expression level changes of GRFs, miR396a target genes, are expected. In addition, we expected to see phenotypes changes of poplar in growth and development. 


\section{Chapter 2 Materials and Methods}

\subsection{Plasmid construction, plant transformation and growth condition}

The PCAMBIA1300STTM396a construct was made and introduced into Agrobacterium tumefaciens EHA105 by Dr. Liu (Liu, 2017). The STTM396a was made based on mature sequence of miR396a as shown in Figure 1.3, group A. and can be expressed as the transcript shown in Figure 2.1. The Agrobacterium strain containing STTM396a was cultured with Rifampicin and Kanamycin as selection markers. Positive colonies were verified for an inserted DNA by PCR using B4 and B5 primers before using Agrobacterium for plant transformation. In this study, Populus tremula $\times$ Populus alba clone 717-1B4 was used as wild type (WT) control and to generate transgenic plants. The Agrobacterium carrying STTM396a construct was used to transform wild type poplar plants by leaf disk transformation method previously described by (Tsai et al. 1994; Liu et al., 2012).

Briefly, wild type leaves were cut to allow Agrobacterium infection through several steps. Then, callus formation was induced after growing the infected leaves on callus induction media (CIM) for three to four weeks under light and dark cycle for 16 and 8 hours at $25^{\circ} \mathrm{C}$ in a growth chamber. Next, calluses were moved to shoot induction medium (SIM) and shoot elongation medium (SEM) respectively for three to four weeks each to allow shoots to grow and elongate. Each shoot that grown out from separate callus was considered as an independent line and was moved to root induction medium (RIM) for another three to four weeks. Each line that started rooting was propagated on Murashige and Skoog (MS) media for another four weeks. Hygromycin $(10 \mu \mathrm{g} / \mathrm{ml})$ was used as a selection marker for STTM plants.

Then, DNA of both wild type and transgenic plants were isolated and transgene insertion was verified by PCR using the B4 and B5 primers before moving plants from artificial media to soil. Plants were acclimatized in soil and 
watered as needed for two to three weeks before moving to greenhouse for further experiments. In greenhouse, plants were grown under light and dark cycle for 16 and 8 hours, respectively at $23-28{ }^{\circ} \mathrm{C}$, watered every day and applied regular fertilizer supply every two weeks.

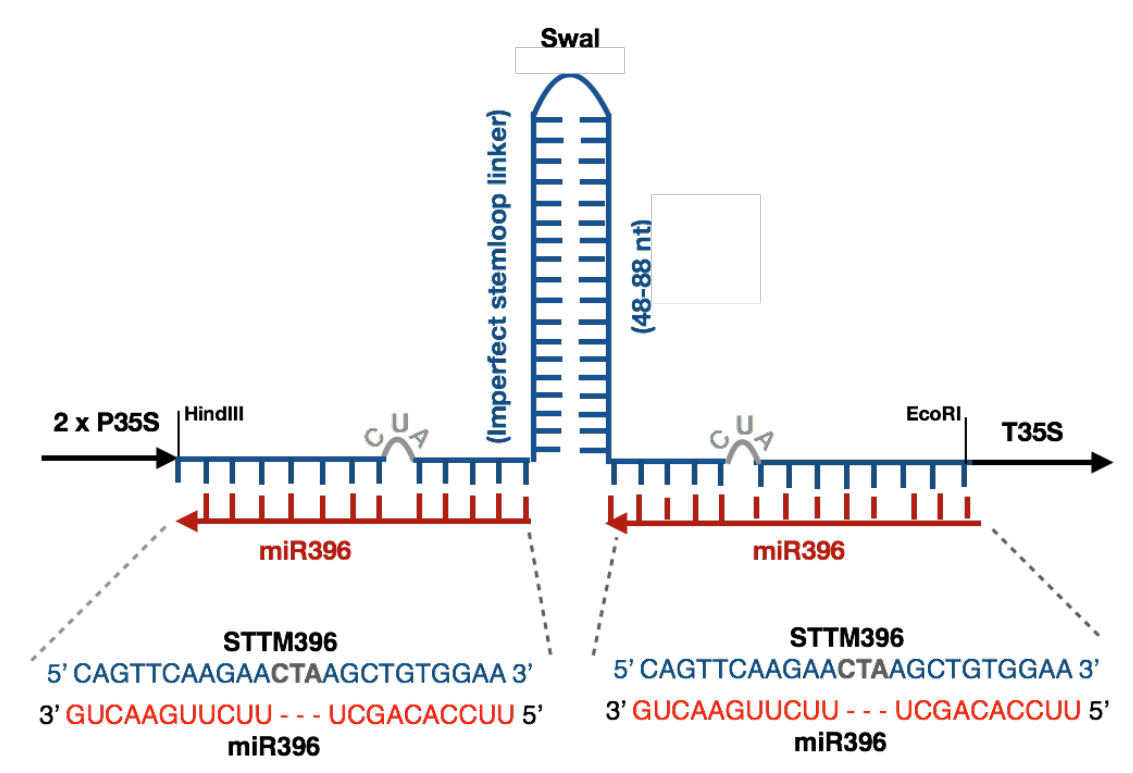

Figure 2.1: Transcript of STTM396a.

The STTM396 transcript binds with miR396a through STTM396a binding sites.

\subsection{Phylogenetic analysis}

The protein sequence of 9 ath-GRFs and 19 ptc-GRFs were obtained from plant transcription factor database or PlantTFDB (http://planttfdb.cbi.pku.edu.cn). Then all 28 protein sequences were aligned using ClustalX 2.1 (Larkin et al., 2007) before performing phylogenetic tree analyses. A phylogenetic tree was generated with OMEGA 7.0 (Hall, 2013) program using neighbor-joining (NJ) method and 1,000 replicates of bootstrapping test.

\subsection{Total RNA isolation and stem-loop RT-qPCR}

PsRNATarget (https://plantgrn.noble.org/psRNATarget/analysis) was used to select predicted miR396 target genes for RT-qPCR. All 19 ptc-GRFs were 
ranked by setting at 25 for maximum energy to unpair the target site (UPE) and at 3 for maximum expectation for complementarity. The first five GRFs including GRF07 (Potri.003G100800.1), GRF10 (Potri.006G143200.1), GRF17 (Potri.015G006200.1), GRF18 (Potri.018G065400.1) and GRF06 (Potri.003G065000.1) were chosen to represent predicted ptc-miR396 target genes.

Leaves were collected and stored in liquid nitrogen. Total RNA was isolated using TRIzol (Invitrogen, USA) method. The stem- loop RT primers and qPCR primers for miR396 were designed according to the method developed by Kramer (Kramer, 2011). The $1 \mu \mathrm{g}$ total RNA was reverse transcribed using a high-capacity cDNA Archive kit (Applied Biosystems, USA). Applied Biosystems step one machine was applied to perform stem-loop qPCR for miRNA (Varkonyi-Gasic et al., 2007) and qPCR for mRNA using SYBR green PCR master mix (Applied Biosystems, USA). Actin was used as an internal control for qPCR. All primers are listed in Table 2.1. All gene expression analysis was performed using three biological replicates or more.

Table 2.1: Primers for real-time PCR and stem-loop real-time PCR.

\begin{tabular}{ll}
\hline Primer name & Sequence \\
\hline miR396 Forward & ACACTCCAGCTGGGTTCCACAGCTTTCTT \\
\hline miR396 Reverse & CTCAACTGGTGTCGTGGAGTCGGCAATTC \\
& AGTTGAGCAGTTCAA \\
\hline Potri.003G100800.1GRF07 Forward & GCAGAGGGTATCCAACTAATC \\
\hline Potri.003G100800.1GRF07 Reverse & ATGGAGAGACCTGTAGTCTTTA \\
\hline Potri.006G143200.1 GRF10 Forward & ATCCCTCCCGATCTTCTTT \\
\hline Potri.006G143200.1 GRF10 Reverse & TCCTTCCCAAACCCATCT \\
\hline Potri.015G006200.1 GRF17 Forward & GTGCCTCCTCATCTTCTAATC \\
\hline Potri.015G006200.1 GRF17 Reverse & CCTCCATTTCTTCCCATCTG \\
\hline Potri.018G065400.1GRF18 Forward & CGGAATTGGTCTGTTACCTC \\
\hline Potri.018G065400.1GRF18 Reverse & GTTCGTCACCTCCTCTTTC \\
\hline Potri.003G065000.1GRF06 Forward & GACTCTTTCCTCATCAACCTAC \\
\hline Potri.003G065000.1 GRF06 Reverse & AGCACCTCCATTTCTTTCC \\
\hline Stem-loop universal Reverse & CTGGTGATTTCAGCGTACCGAA \\
\hline B4 & AACCTCCTCGGATTCCATTGCCCAGC \\
\hline B5 & CCTTGTCTTCCTCCTTCCTTCCGTCC \\
\hline Actin Forward & ATGGCCGATTCTGAGGATATTCAG \\
\hline Actin Reverse & TCTGCCCCATTCCAACCATGACA \\
\hline
\end{tabular}




\subsection{Microscopy}

Wild type and transgenic leaves were cross-cut at the middle into $0.4 \mathrm{~cm}$ pieces and immersed in cold fixative solution containing formaldehyde, acetic acid, and ethanol overnight at $4{ }^{\circ} \mathrm{C}$. Then, fixed samples were dehydrated and embedded in wax. Next, five micrometers thick section were crosscut consecutively with 100 micrometers between level by microtome and mounted on slides (performed by Sharon Juntilla at Histotech lab, Portage Hospital, Hancock). Slides were dewaxed with xylene and ethanol series before stained with safranin and observed under light microscope (Adapted from Johansen, 1940). To investigate cell proliferation and cell expansion change in cellular level, cell size and cell number were measured within the same four areas of leaves cross section for three pictures each using ImageJ program (https://imagej.nih.gov/ij/).

\subsection{Growth measurements}

After plants were moved to the greenhouse for one week, growth data including estimated height $(\mathrm{cm})$, estimated stem girth $(\mathrm{mm})$, leaf count $(\mathrm{n})$, and leaf length $(\mathrm{cm})$ were measured every week.

\subsection{Statistical analysis}

In this study, SPSS 25.0 software (https://www.ibm.com/analytics/spssstatistics-software) was used to conduct statistical significance analysis of the data. Student T-test was used to analyze two data sets. One asterisk ( $\mathrm{p} \leq 0.05)$, two asterisks ( $p \leq 0.01)$, and three asterisks ( $p \leq 0.001)$ were displayed. 


\section{Chapter 3 Results}

\subsection{Phylogenetic tree of GRFs}

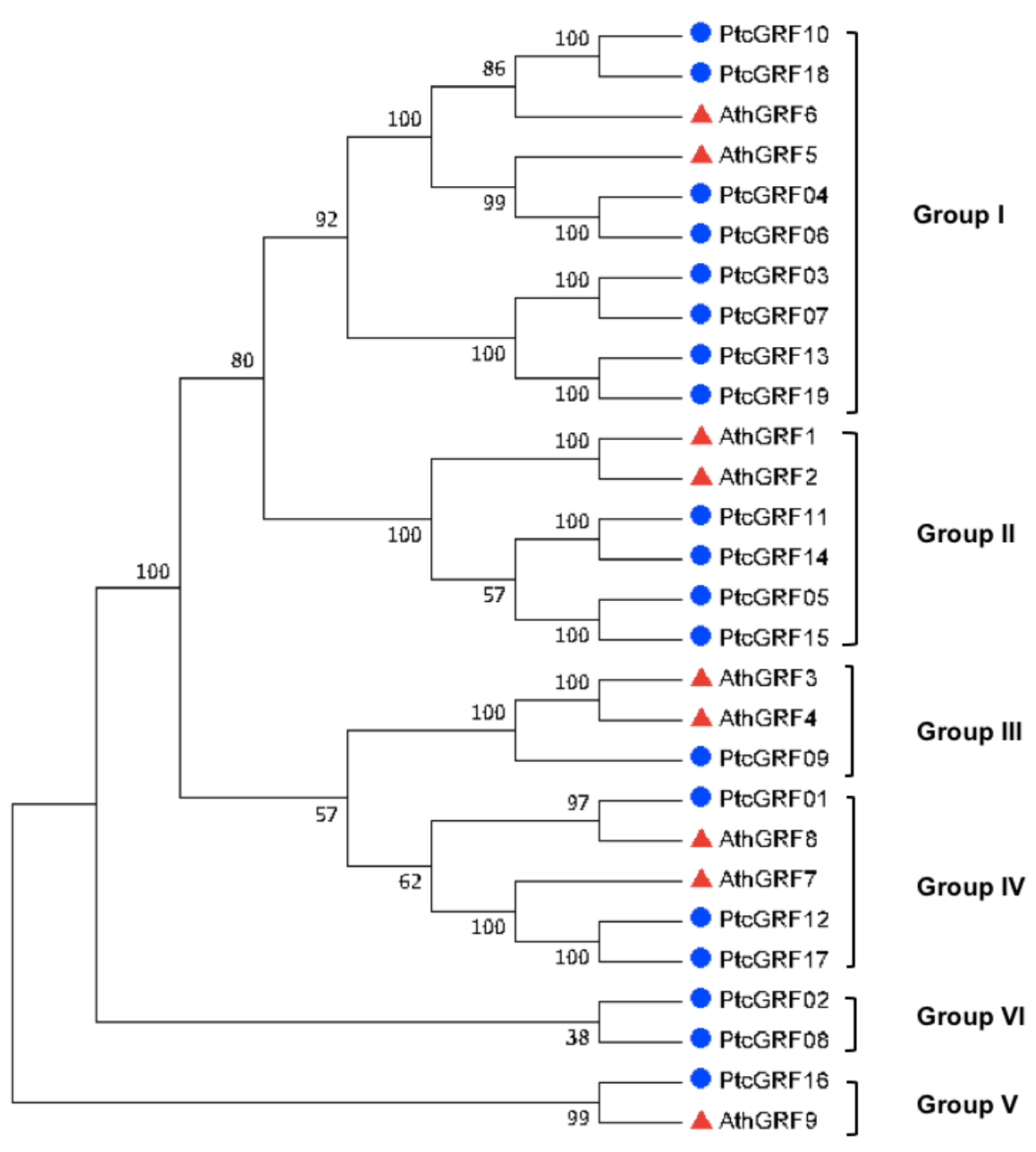

Figure 3.1: Phylogenetic tree of AthGRFs and PtcGRFs.

Predicted GRF protein sequences from Arabidopsis (Ath-GRF) and poplar (Ptc-GRF) indicated PtcGRF19 and AthGRF9 are outgroup. GRFs can be divided into 6 groups. The phylogenetic tree also suggested that some of GRFs are identical including PtcGRF10 and PtcGRF18, PtcGRF04 and PtcGRF06, PtcGRF03 and PtcGRF07, PtcGRF13 and PtcGRF19, PtcGRF11 and PtcGRF14, PtcGRF05 and PtcGRF15, PtcGRF12 and PtcGRF17, PtcGRF02 and PtcGRF08 in poplar. In Arabidopsis, there are a few GRFs that are identical including 
AthGRF1 and AthGRF2 and AthGRf3 and AthGRF4. These could be a result from genome duplication in poplar. Also, those identical GRFs might play redundancy roles in plants. According to the phylogenetic tree, AthGRFs and PtcGRFs can be classified into six group including group I (AthGRF6, AthGRF5, PtcGRF10, PtcGRF18, PtcGRF04, PtcGRF06, PtcGRF03, PtcGRF07, PtcGRF13, PtcGRF19), group II (AthGRF1, AthGRF2, PtcGRF11, PtcGRF14, PtcGRF05, PtcGRF15), group III (AthGRF3, AthGRF4, PtcGRF09), group IV (PtcGRF01, AthGRF8, AthGRF7, PtcGRF12, PtcGRF17), group V (PtcGRF02, PtcGRF08), group VI (PtcGRF16, AthGRF9). According to those six group, group VI including PtcGRF16 and AthGRF9 is the out group suggesting they might play similar role in poplar and Arabidopsis. Group V includes only PtcGRF02 and PtcGRF08 implying that they might play some specific role only in poplar but not in Arabidopsis.

\subsection{MiR396 was effectively knocked down in poplar by STTM}

MiR396 was knocked down effectively in poplar by STTM. STTM396 resulted in dramatically decreased relative expression level of miR396 in four lines of STTM396 plants, \#3 \#5 \#6 \#9, compared to one line in wild type (Figure 3.2 A). The predicted miR396 target genes showed GRF07 up-regulation (Figure $3.2 \mathrm{C}$ ), down-regulation of GRF06 and GRF18 (Figure 3.2 B and 3.2 F). Besides, another two targets genes, GRF10 and GRF17, were barely expressed in both wild type and STTM plants (Figure 3.2 D and 3.2 E). 


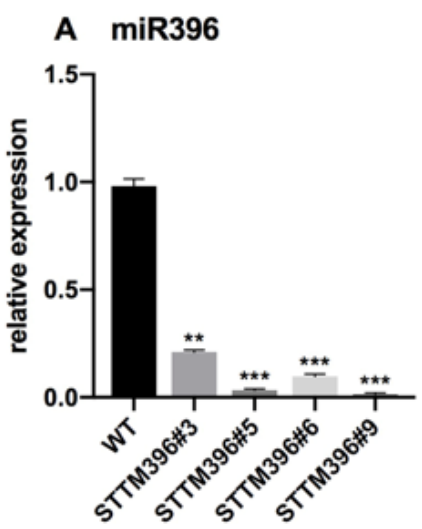

D GRF10

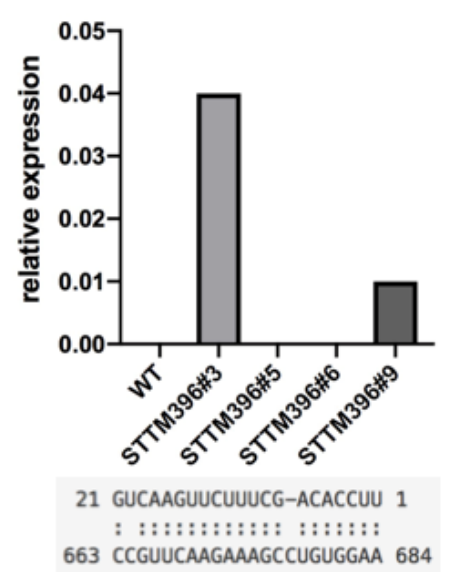

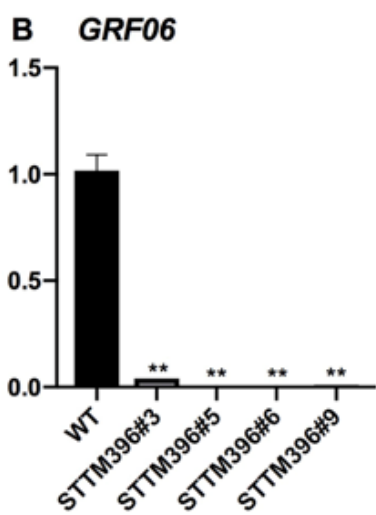

21 GUCAAGUUCUUUCG-ACACCUU 1

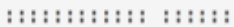
345 UCGUUCAAGAAAGCCUGUGGAA 366

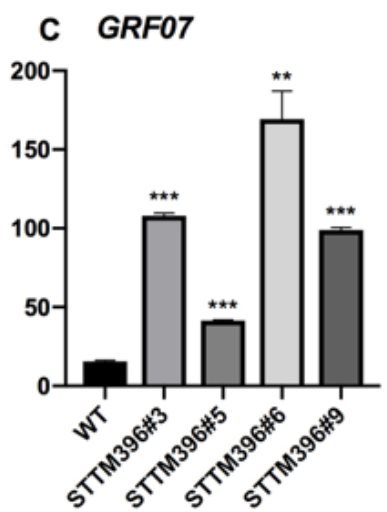

21 GUCAAGUUCUUUCG-ACACCUU 1

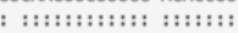
400 CCGUUCAAGAAAGCCUGUGGAA 421
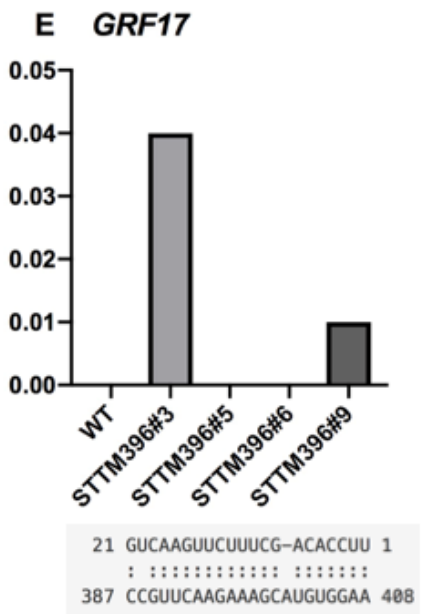

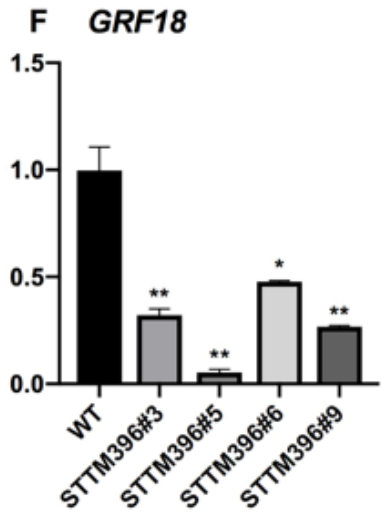

21 GUCAAGUUCUUUCG-ACACCUU 1

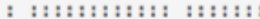
959 CCGUUCAAGAAAGCCUGUGGAA 980

Figure 3.2: MiR396 was effectively knocked down in poplar by STTM. (A-F) RT-qPCR analysis of miR396 and five predicted target genes in WT and four independent lines of STTM plants. Binding sites between miR396 and its target genes, which are in coding region of QLQ domain, are shown. Asterisk displayed statistically significant difference applying student paired t-test $\left({ }^{*} p \leq 0.05\right.$, $\left.{ }^{* *} \mathrm{P} \leq 0.01,{ }^{* * *} \mathrm{p} \leq 0.001\right)$. The error bars indicated mean $\pm \mathrm{SD}$.

\subsection{Blockage of miR396 induced taller plants with larger leaves}

After plants were moved to the greenhouse, growth measurement was recorded every week. Estimated leaf length from base to tip of the first two leaves from the bottom of wild type and five lines of transgenic plants were tracked. 
STTM396 plants showed significantly larger leaf length in both positions compared to one in wild type (Figure 3.3-3.4 and 3.6 B).

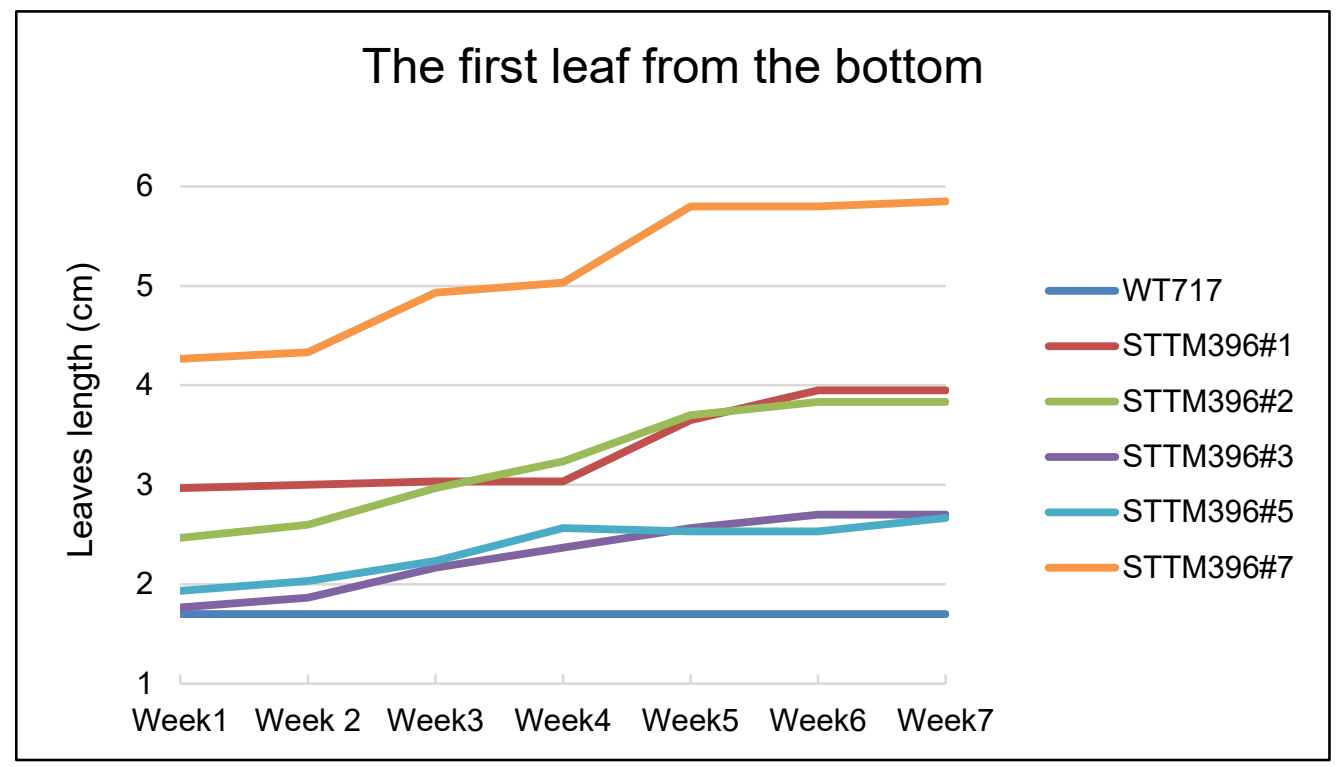

Figure 3.3: Length of the first leaf from the bottom in centimeters $(\mathrm{cm})$. Comparison between wild type and STTM396 plant leaves over seven weeks.

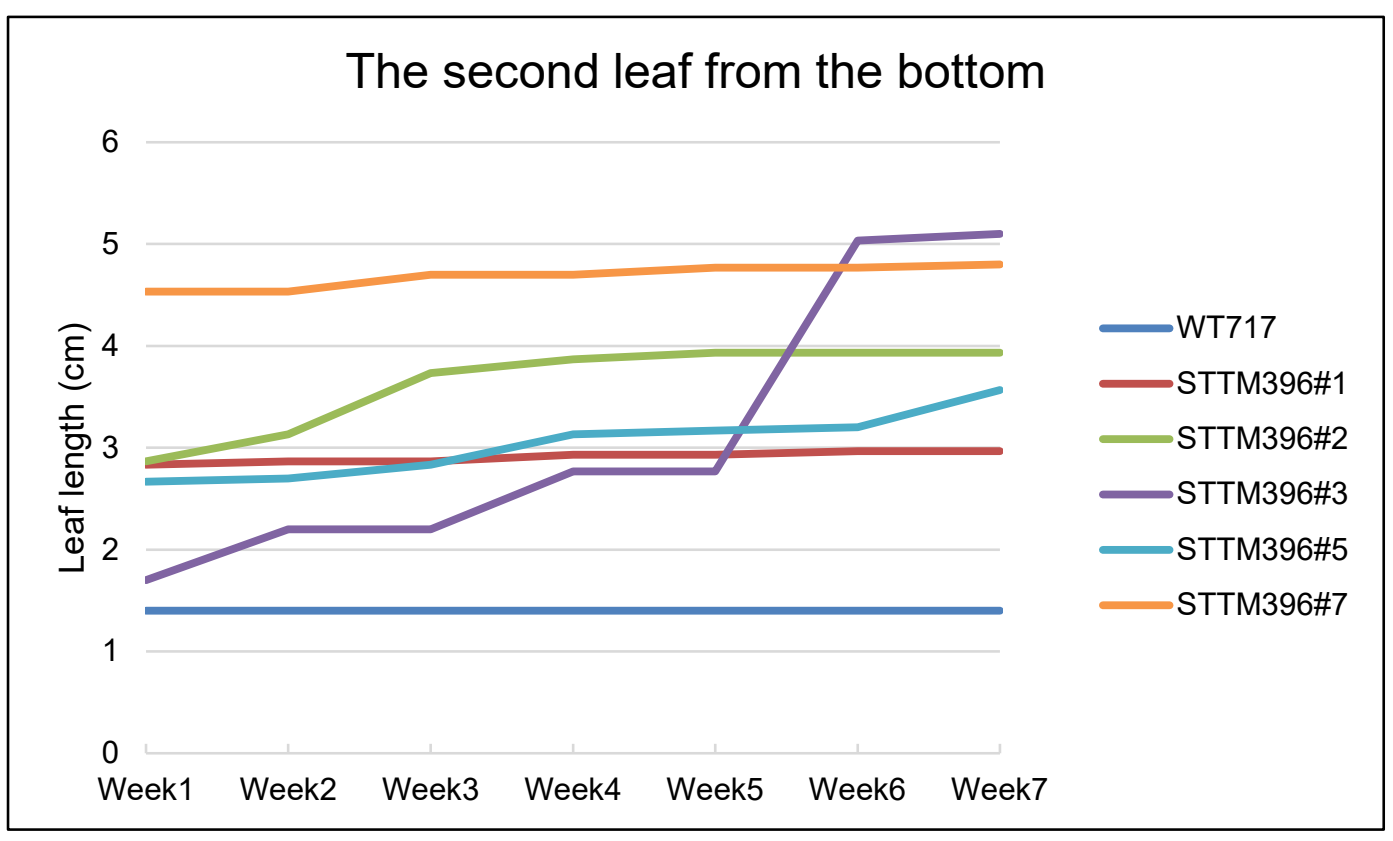

Figure 3.4: Length of the second leaf from the bottom in centimeter $(\mathrm{cm})$. Comparison between wild type and STTM396 plants over seven weeks shown. 


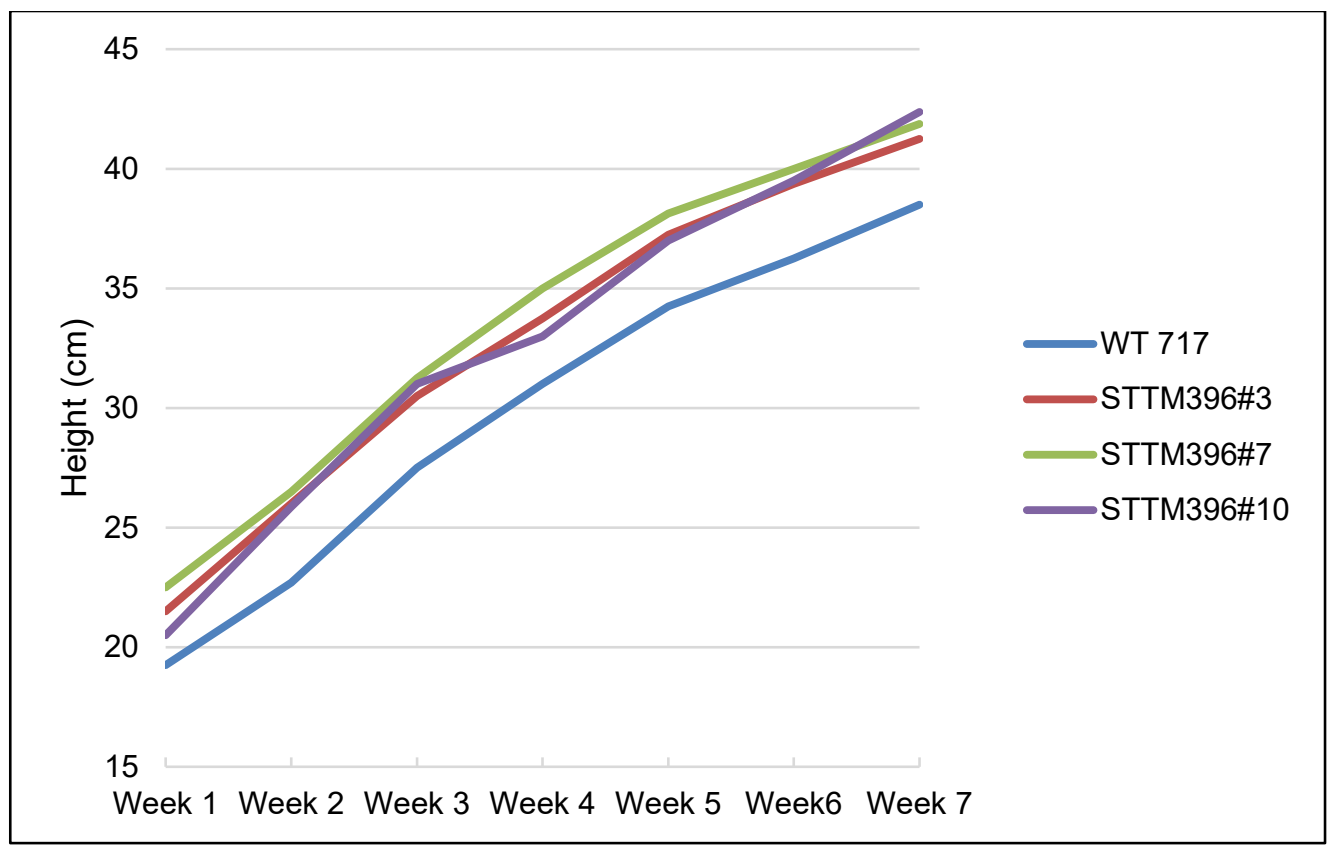

Figure 3.5: Estimated height in centimeter $(\mathrm{cm})$ of wild type and STTM396 plants over seven weeks.
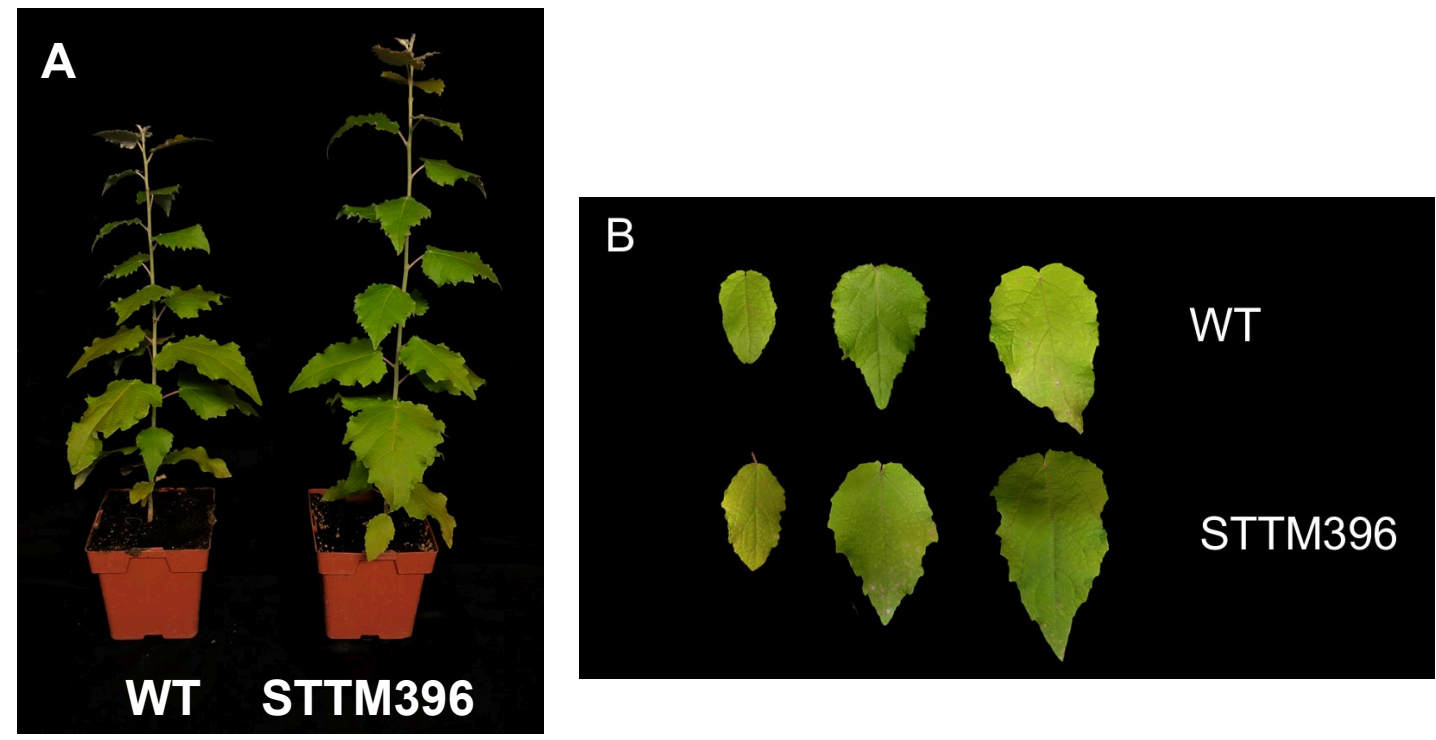

Figure 3.6: Comparison plant stature $(A)$ and leaf length $(B)$ between wild type and STTM396 plant.

Remarkably, transgenic plants were notably taller but had smaller stem girth than in wild type plants (Figure 3.5, 3.6 A and 3.7). To explain this phenomenon, more lines of transgenic plants should be examined. Besides, leaf count data 
showed inconsistent trends in STTM plants (Figure3.8). More lines of transgenic plants are also required to conclude this result.

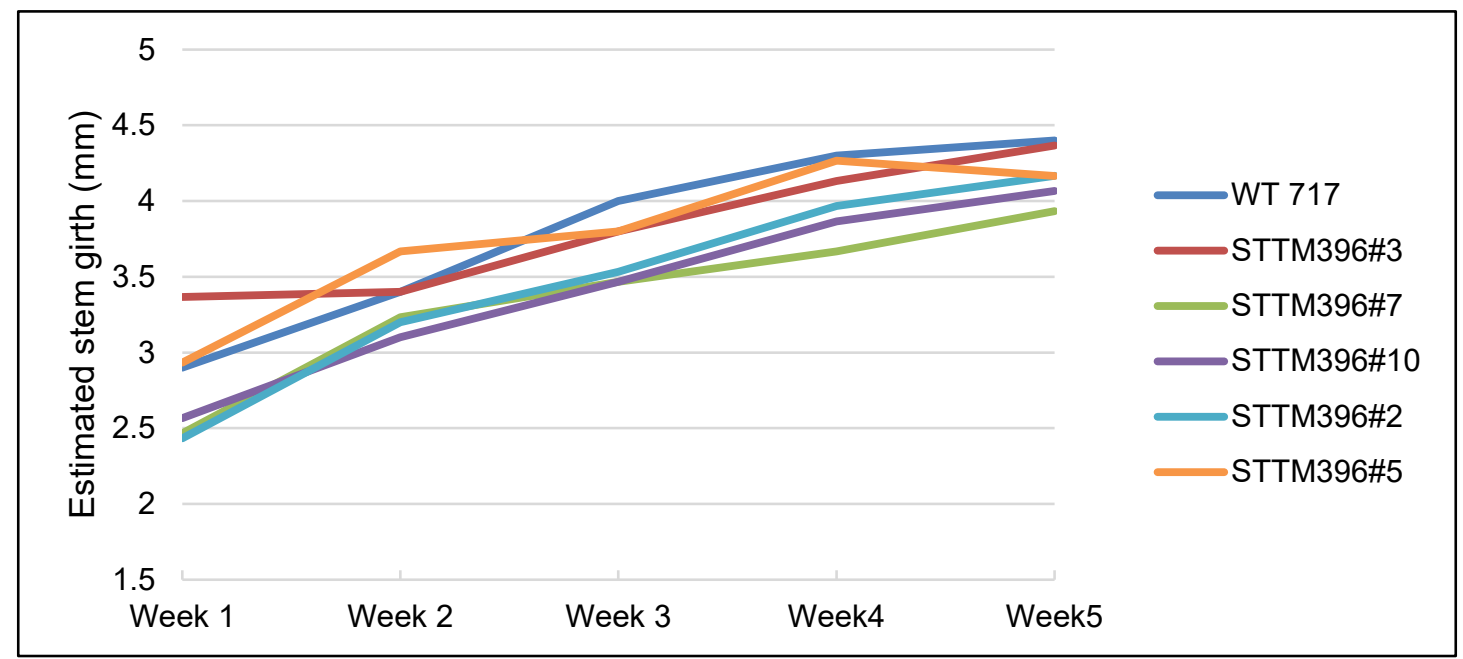

Figure 3.7: Estimated stem girth in millimeter $(\mathrm{mm})$ of wild type and STTM396 plants over seven weeks.

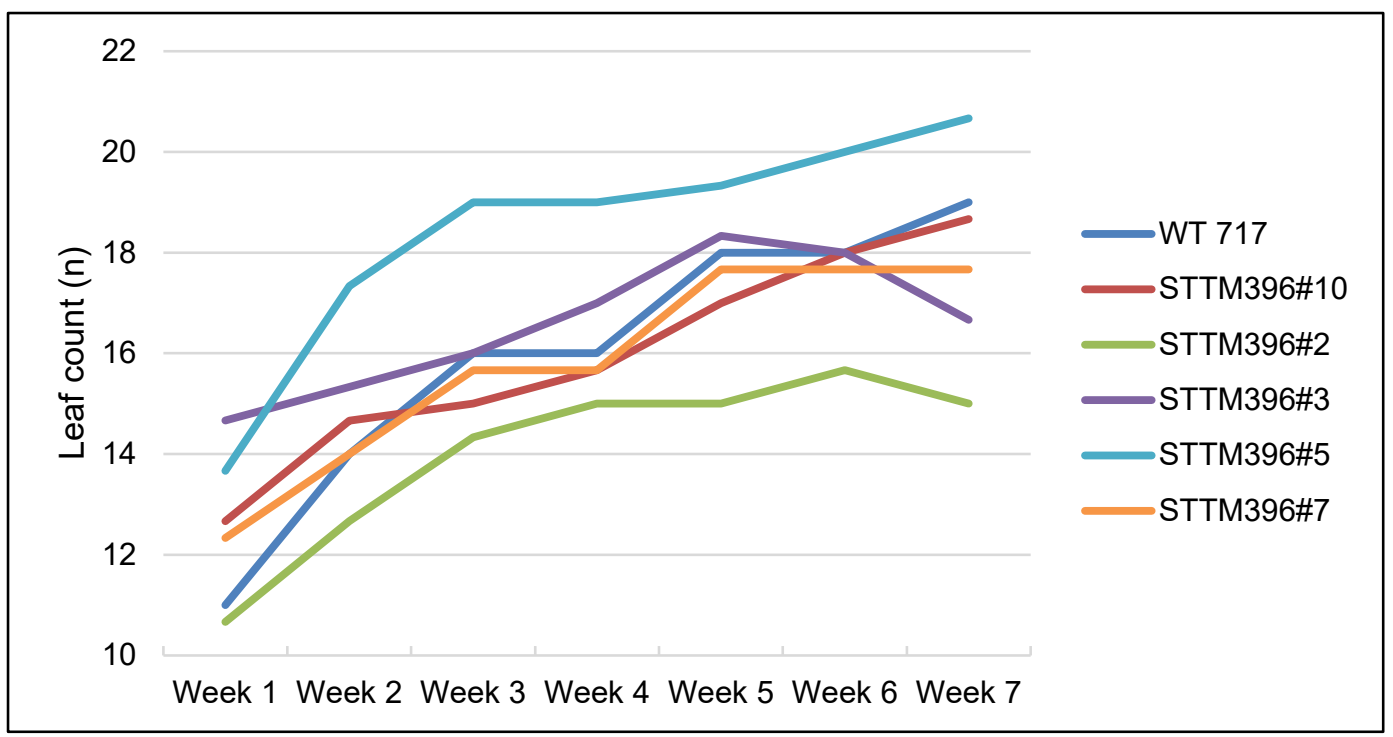

Figure 3.8: Leaf count (n) of wild type and STTM396 plants over seven weeks. 


\subsection{STTM396 plants have altered leaf cell size and cell number}

To investigate cell proliferation and cell expansion change in cellular level, cross-section of wild type and STTM396 leaves were observed under bright field microscope. STTM396 resulted in larger cell size and smaller cell number in all four areas (Figure 3.9, 3.10 and 3.11) suggesting that altered leaves size was a result from cell expansion rather than cell proliferation.
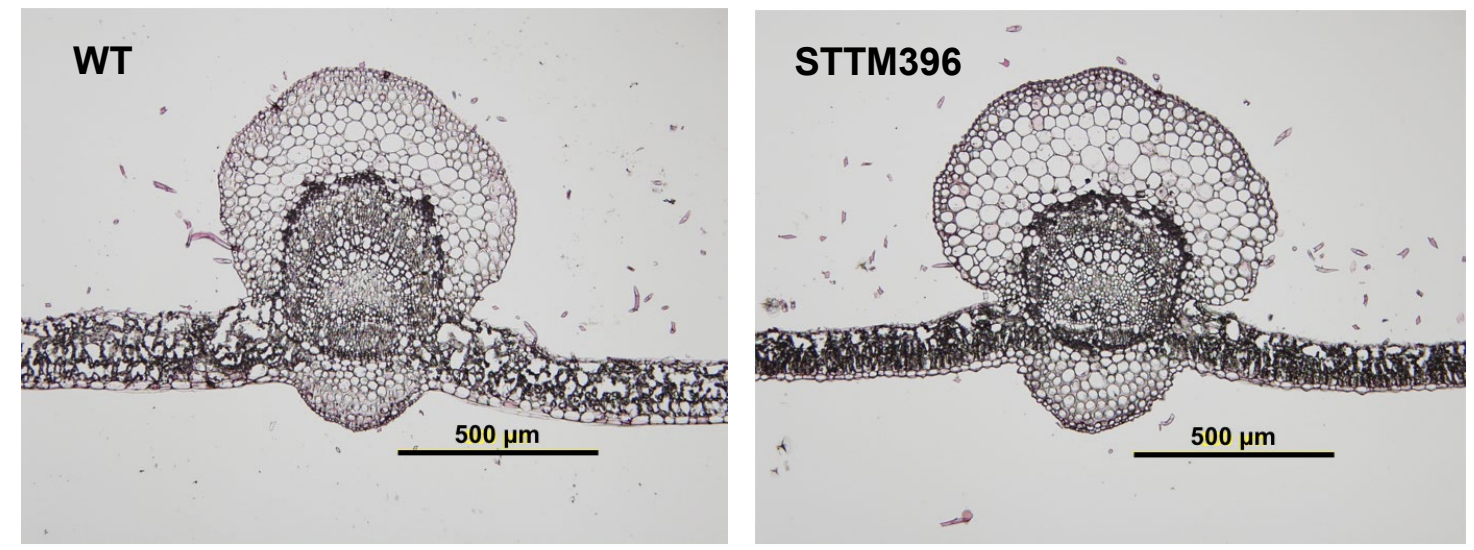

Figure 3.9: Transverse section of wild type and STTM396 leaves, particularly at mid vein region.
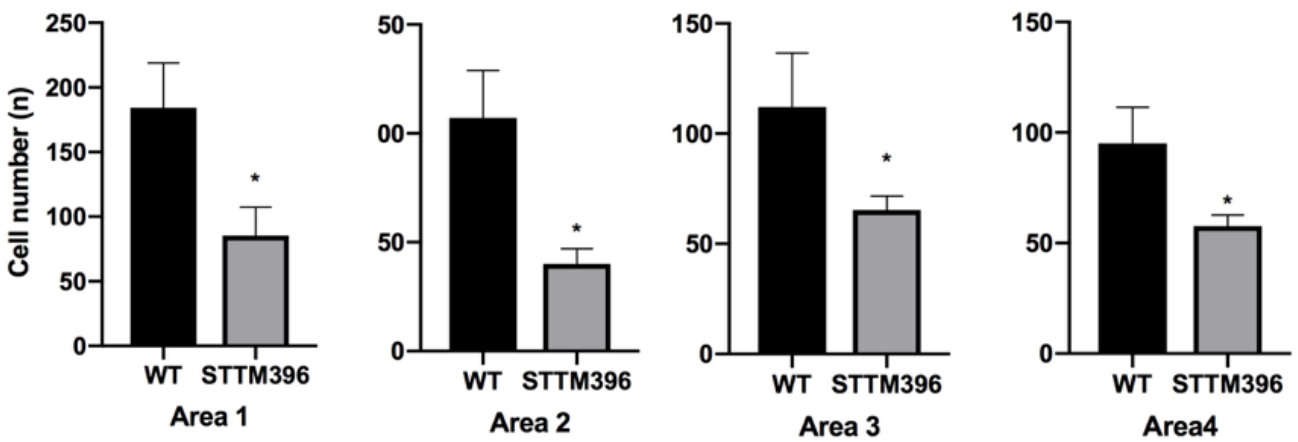

Figure 3.10: STTM396 resulted in smaller cell number in STTM leaves. Cell number measurement in WT and STTM leaves. Asterisk displayed statistically significant difference applying student paired t-test $\left({ }^{*} p \leq 0.05\right.$, $\left.{ }^{* *} \mathrm{P} \leq 0.01,{ }^{* * *} \mathrm{p} \leq 0.001\right)$. The error bars indicated mean $\pm \mathrm{SD}$. 

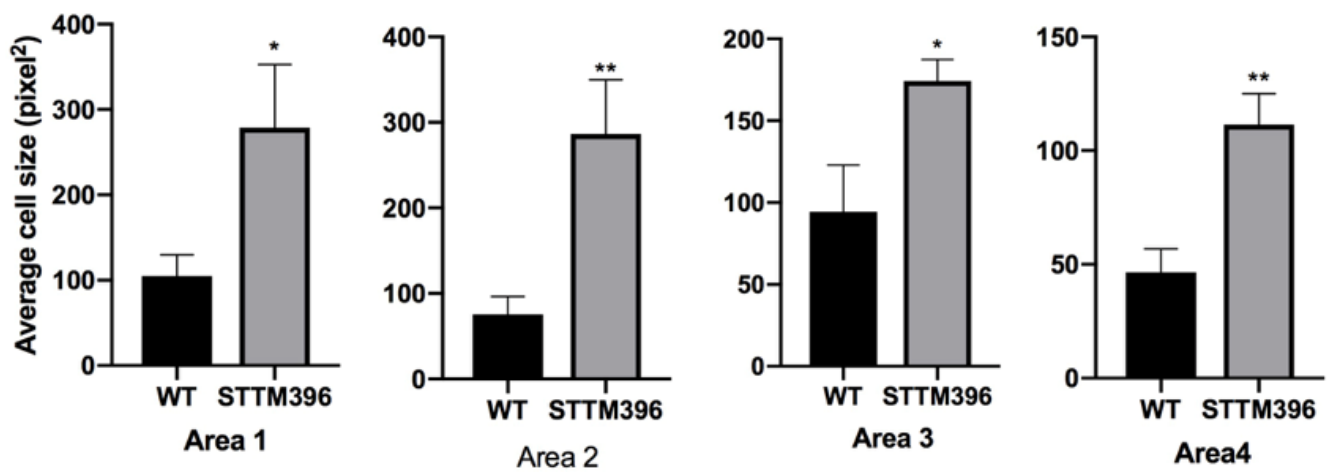

Figure 3.11: STTM396 resulted in larger cell size in STTM leaves.

Cell size measurement in WT and STTM leaves. Asterisk displayed statistically significant difference applying student paired t-test $\left({ }^{*} p \leq 0.05,{ }^{* *} \mathrm{P} \leq 0.01\right.$, $\left.{ }^{* * *} p \leq 0.001\right)$. The error bars indicated mean \pm SD. 


\section{Chapter 4 Discussion}

\subsection{MiR396 was effectively knocked down in poplar by STTM}

MiR396 is one of the highly conserved miRNAs among plant species. Mature sequence of miR396a in Arabidopsis, rice, and maize and miR396a and miR396b in poplar are identical (http://www.mirbase.org). Their targets are members of GRFs gene family, which are plant-specific transcription factors. Poplar has 19 GRFs, whereas Arabidopsis, rice, and maize has 9, 12, and 14 GRFs respectively. In addition, GRFs genes are distributed unevenly in poplar genome, mostly on chromosome 01 and 14 (Kim et al., 2003; Choi et al., 2004; Zhang et al., 2008).

STTM has been used to knockdown small RNAs successfully in fungi, animals, and several plants species (Wang et al., 2019; Tang et al., 2012; Yan et al., 2012). Here, we show that STTM can also disrupt miRNA functions effectively in poplar, which represents a tree species. Remarkably, STTM396 resulted in 7898\% suppression of miR396 expression levels (Figure 3.2 A).

\subsection{Blockage of miR396 induced taller plants with larger leaves}

STTM396 produced taller transgenic plants (Figure 3.6A and 3.7) with larger leaves (Figure 3.6 B, 3.7 and 3.8). These results suggest that ptc-miR396 might be negatively playing a role in leaf growth and development of poplar. This conclusion is consistent with other studies in Arabidopsis and tomato. (LiuKanpp et al., 2009; Cao et al., 2016) suggesting that miR396 as a negative regulator of plant growth.

\subsection{STTM396 plants have altered leaf cell size and cell number}

Furthermore, organ size is basically determined by cell expansion and/or cell proliferation (Rodriguez et al., 2016). To understand causes of organs enlargement, leaf histology was examined. Leaf transverse sections at the midrib 
region showed larger cell sizes but smaller cell number in STTM396 leaves as compared to wild type control (Figure 3.9-3.11). These results indicate that the leaf enlargement may be a result of cell expansion rather than cell proliferation. In stem, STTM396 plants showed increased in height but reduced girth than control plants. These could be resulted from cell expansion or cell elongation as in rice and Arabidopsis. Previous studies provided some clues about miR396 regulatory functions through its target genes, GRFs. For example, Van der Knaap et al. (2000) suggested that osa-GRF1 may be involved with stem elongation in rice. Also, suppression of osa-GRFs resulted in dwarf plants. (Kuijt et al., 2014).

However, processes of stem growth and development in tree species are likely to be more complicated than monocot plants and other herbaceous dicot plants. Furthermore, a careful histological study of transgenic poplar stems expressing STTM396 is required to explain these phenotypes. Therefore, the regulation of cell proliferation and expansion by miR396 is complicated and might be different among plant species and even in different tissues.

\subsection{Expression level of GRFs changed in STTM396 plants}

Mir396 target genes expression analysis in transgenic poplars expressing STTM396 showed that GRF07 was up-regulated but GRF06 and GRF18 were down-regulated in STTM plants. These results suggested that miR396 normally plays a role as negative regulator of growth and cell expansion through GRF07 suppression. Additionally, GRF06 and GRF18 may be not only regulated by miR396 but also controlled by another gene or another miRNA as shown in Figure 4.1 in a much stronger manner. Also, qRT-PCR analysis suggests that GRF10 and GRF17 are barely expressed in leaves tissues suggesting that these two genes may be expressed in other tissues for example stem instead of leaves. Similarly, bra-GRFs were expressed in a tissue-specific manner in Chinese cabbage. BraGRF1, 4, 6, 8, 10, 15, and 17 were expressed in young leaf higher than in other tissues (Wang, 2014). However, another 14 ptc-GRFs are required to be studied 
for a better understanding of role of various GRFs in poplar growth. Also, overexpression of GRF07 might help to confirm the roles of GRF07 in poplars.

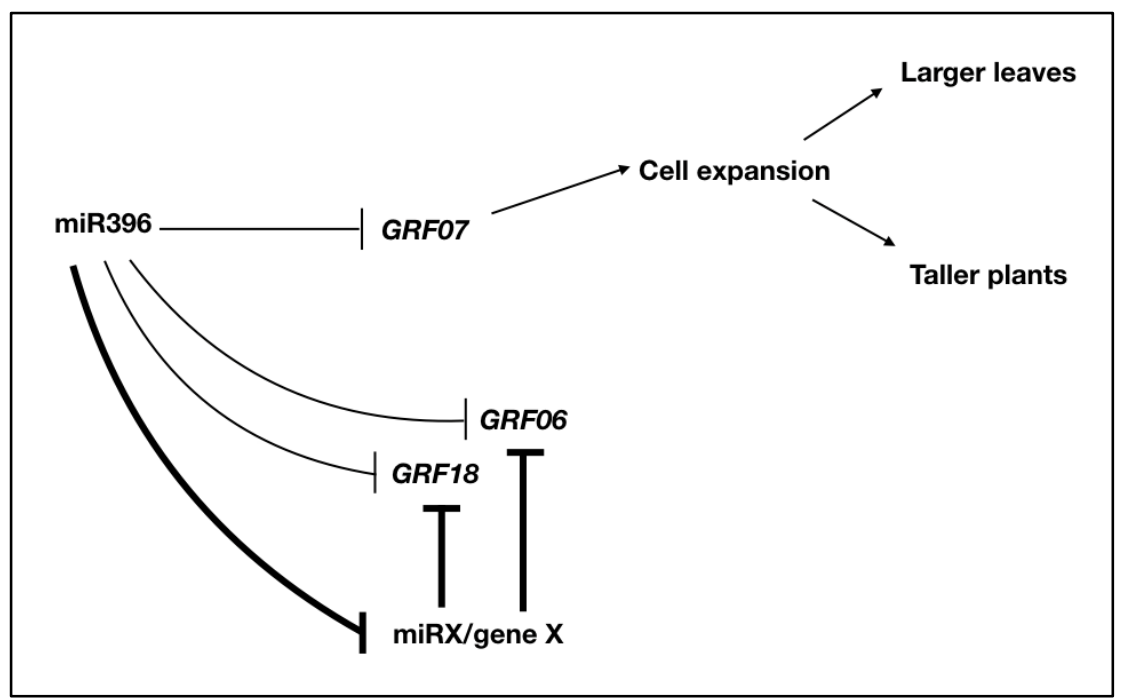

Figure 4.1: Proposed schematic relations between miR396 and GRFs affecting -1 growth and deve in poplar. 
and development. Furthermore, ptc-miR396 mediate ptc-GRFs expression is a complex network. They might be expressed and function in tissue-specific manner.

\subsection{Future studies}

In the future, stem GRFs should be studied for a better understanding of interrelationship between miR396 and GRFs in poplars. To complete the whole picture of miR396 and ptc-GRFs functions, the rest of the 14 GRFs should be also studied in both leaves and stems of transgenic STTM396 poplar plants. Furthermore, importance of miR396 and ptc-GRFs in reproductive organs should also be explored in the future. This could be done via their ectopic expression in other plant model species such as Arabidopsis or tobacco because flowering in poplars takes many years. 


\section{References}

Axtell, M.J., Westholm, J. O., and Lai, E. C. 2011. 'Vive la différence: biogenesis and evolution of microRNAs in plants and animals', Genome Biology, 12: 221.

Bartel, D. P. 2004. 'MicroRNAs: Genomics, Biogenesis, Mechanism, and Function', Cell, 116: 281-97.

Baucher, M., Moussawi, J., Vandeputte, O. M., Monteyne, D., Mol, A., Pe'rezMorga, D., and Jaziri, M. E. 2013. 'A role for the miR396/GRF network in specification of organ type during flower development, as supported by ectopic expression of Populus trichocarpa miR396c in transgenic tobacco', plant biology, 15: 892-98.

Cao, D., Wang, J., Ju, Z., Liu, Q., Li, S., Tian, H., Fu, D., Zhu, H., Luo, Y., and Zhu, B. 2016. 'Regulations on growth and development in tomato cotyledon, flower and fruit via destruction of miR396 with short tandem target mimic', Plant Sciences, 247: 1-12.

Choi, D., Kim, J. H., and Kende, H. 2004. 'Whole genome analysis of the OsGRF gene family encoding plant-specific putative transcription activators in rice ', Plant \& Cell Physiology, 45: 897-904.

Damodharan, S., Zhao, D., and Arazi., T. 2016. 'A common miRNA160-based mechanism regulates ovary patterning, floral organ abscission and lamina outgrowth in tomato', The Plant Journal, 86: 458-71.

Dong, C.H., and Pei, H. 2014. 'Over-expression of miR397 Improves Plant Tolerance to Cold Stress in Arabidopsis thaliana ', plant biology, 57: 209.

Gao, F., Wang, K., Liu, Y., Chen, Y., Chen, P., Shi, Z., Luo, J., Jiang, D., Fan, F., Zhu, Y., and Li, S. 2015. 'Blocking miR396 increases rice yield by shaping inflorescence architecture', Nature Plants, 2: 1-9.

Gasic, E. V., Wu, R., Wood, M., Walton, E. F., and Hellens, R. P. 2007. 'Protocol: a highly sensitive RT-PCR method for detection and quantification of microRNAs', Plant Methods, 3: 12.

Griffiths-Jones, S., Grocock, R.J., Van Dongen, S., Bateman, A., and Enright, A.J. 2006. 'miRBase: microRNA sequences, targets and gene nomenclature', Nucleic Acids Research, 34: 140-44.

Hall, B. G. 2013. 'Building Phylogenetic Trees from Molecular Data with MEGA', Molecular Biological Evolution, 30: 1229-35. 
Jia, X., Ding, N., Fan, W., Yan, J., Gu, Y., Tang, X., Li, R., and Tang., G. 2015. 'Functional plasticity of miR165/166 in plant development revealed by small tandem target mimic', Plant Science, 2015: 11-21.

Johansen, D. A.. 1940. Plant Microtechnique. McGraw-Hill Book Co., New York.

Khan, G. A., Declerck, M., Sorin, C., Hartmann, C., Crespi, M., and LelandaisBrie're, C. 2011. 'MicroRNAs as regulators of root development and architecture ', Plant Molecular Biology, 77: 47-58.

Khraiwesh, B., Zhu, J.K., and Zhu, J. 2012. 'Role of miRNAs and siRNAs in biotic and abiotic stress responses of plants', Biochim Biophys Acta, 1819: 13748.

Kim, J. H., Choi, D., and Kende, H. 2003. 'The AtGRF family of putative transcription factors is involved in leaf and cotyledon growth in Arabidopsis', The Plant Journal, 36: 94-104.

Kim, J. Y., Kwak, K. J., Jung, H. J., Lee, H. J., and Kang, H. 2010. 'MicroRNA402 Affects Seed Germination of Arabidopsis thaliana Under Stress Conditions via Targeting DEMETER-LIKE Protein3 mRNA', Plant Cell Physiology, 5: 1079-83.

Kramer, M. 2011. 'STEM-LOOP RT-qPCR for miRNAS', Current Protocols in Molecular Biology, 95.

Kuijt, S.J., Greco, R., Agalou, A., Shao, J., Hoen, C.C., Övernäs, E., Osnato, M., Curiale, S., Meynard, D., Van Gulik, R., De Faria Maraschin, S., Atallah, M., De Kam, R.J., Lamers, G.E.M., Guiderdoni, E., Rossini, L., Meijer, A.H., and Ouwerkerk, P.B.F. 2014. 'Interaction between the GROWTH-REGULATING FACTOR and KNOTTED1-LIKE HOMEOBOX Families of Transcription Factors', Plant Physiology, 164: 1952-66.

Kumar, K.S. "Manipulation of Genes Involved in Secondary Cell Wall Development During Wood Formation in Poplar", Open Access Dissertation, Michigan Technological University, 2018. https://digitalcommons.mtu.edu/etdr/591

Larkin, M.A., Blackshields, G., Brown, N.P., Chenna, R., McGettigan, P.A., and H. McWilliam, Valentin, F., Wallace, I. M., Wilm, A., Lopez, R., Thompson, J.D., Gibson, T.J., and Higgins, D.G. 2007. 'Clustal W and Clustal X version 2.0', Bioinformatics, 23: 2947-48.

Li, C., and Zhang, B. 2016. 'MicroRNAs in Control of Plant Development', Journal of cellular physiology, 231: 303-13. 
Liang, G., He, H., Li, Y., Wang, F., and Yu, F. 2014. 'Molecular Mechanism of microRNA396 Mediating Pistil Development in Arabidopsis', Plant Physiology, 164: 249-58.

Liu, D., Song, Y., Chen, Z., and Yu. D. 2009. 'Ectopic expression of miR396 suppresses GRF target gene expression and alters leaf growth in Arabidopsis', Physiologia Plantarum, 136: 223-36.

Liu, Haiping, "MODULATION OF MICRORNA EXPRESSIONS IN TOMATO BY SHORT TANDEM TARGET MIMIC AND ARTIFICIAL MIRNAS", Campus Access Dissertation, Michigan Technological University, 2017. https://digitalcommons.mtu.edu/etdr/445

Liu, Y., Xu, F., Gou, J., Al-Haddad, J., Telewski, F.W., Bae, H.J., Joshi, C.P. 2012. 'Importance of two consecutive methionines at the $\mathrm{N}$-terminus of a cellulose synthase (PtdCesA8A) for normal wood cellulose synthesis in aspen', Tree Physiology, 32: 1403-12.

Llave, C., Kasschau, K.D., Rector, M. A., and Carrington J.C. 2002. 'Endogenous and Silencing-Associated Small RNAs in Plants', Plant Cell, 14: 1605-19.

Ma, C., Liu, Y., and He, L. 2009. 'microRNAs - powerful repression comes from small RNAs', Science in China Series C: Life Sciences, 52: 323-30.

Mecchia, M.A., Debernardi, J.M., Rodriguez, R.E., Schommer, C., and Palatnik., J.F. 2013. 'MicroRNA miR396 and RDR6 synergistically regulate leaf development', Mechanisms of Development, 130: 2-13.

Mette, M. F., Van Der Winden, J., Matzke, M., and Matzke, A.J.M. 2002. 'Short RNAs Can Identify New Candidate Transposable Element Families in Arabidopsis', Plant Physiology, 130: 2002.

Omidbakhshfard, M.A., Proost, S., Fujikura, U., and Mueller-Roeber, B. 2015. 'Growth-Regulating Factors (GRFs): A Small Transcription Factor Family with Important Functions in Plant Biology', Molecular Plant, 8: 998-1010.

Pareek, M., Yogindran, S., Mukherjee, S., and Rajam, M. 2015. Plant MicroRNAs: Biogenesis, Functions, and Applications (Plant Biology and Biotechnology).

Park, W., Li, J., Song, R., Messing, J., and Chen, X. 2002. 'CARPEL FACTORY, a Dicer Homolog, and HEN1, a Novel Protein, Act in microRNA Metabolism in Arabidopsis thaliana', Current Biology, 12: 1484-95. 
Peng, T., Qiao, M., Lui, H., Teotia, S., Zhang, Z., Zhao, Y., Wang, B., Zhao, D., Shi, L., Zhang, C., Le, B., Rogers, K., Gunasekara, C., Duan, H., Gu, Y., Tian, L., Nie, J., Qi, J., Meng, F., Huang, L., Chen, Q., Wang, Z., Tang, J., Tang, X., Lan, T., Chen, X., Wei, H., Zhao, Q., and Tang, G. 2018. 'A Resource for Inactivation of MicroRNAs Using Short Tandem Target Mimic Technology in Model and Crop Plants', Molecular Plant, 11: 1400-17.

Reinhart, B.J., Weinstein, E. G., Rhoades, M.W., Bartel, B., and Bartel, D. P. 2002. 'MicroRNAs in plants', Genes \& Development, 16: 1616-26.

Rodriguez, R.E., Mecchia, M.A., Debernardi, J.M., Schommer, C., Weigel, D., and Palatnik, J.F. 2010. 'Control of cell proliferation in Arabidopsis thaliana by microRNA miR396', Development, 137: 103-12.

Rodriguez, R.E., Schommer, C., and Palatnik, J.F. 2016. 'Control of cell proliferation by microRNAs in plants', Current Opinion in Plant Biology, 34: 68-76.

Su, Y., Li, H.G., Wang, Y., Li, S., Wang, H.L., Yu, L., He, F., Yang, Y., Feng, C.H., Shuai, P., Liu, C., Yin, W., and Xia., X. 2018. 'Poplar miR472a targeting NBS-LRRs is involved in effective defence against the necrotrophic fungus Cytospora chrysosperma', Journal of Experimental Botany, 69: 5519-30.

Tang, G., Yan, J., Gu, Y., Qiao, M., Fan, R., Mao, Y., and Tang, X. 2012. 'Construction of short tandem target mimic (STTM) to block the functions of plant and animal microRNAs', Methods, 58: 118-25.

Tsai, C. J., Podila, G. K., and Chiang, V. L. 1994. 'Agrobacterium-mediated transformation of quaking aspen (Populus tremuloides) and regeneration of transgenic plants', Plant Cell Reports, 14: 94-97.

Tuskan, G. A., S. DiFazio, S. Jansson, J. Bohlmann, I. Grigoriev, U. Hellsten, N. Putnam, S. Ralph, S. Rombauts, A. Salamov, J. Schein, L. Sterck, A. Aerts, R. R. Bhalerao, R. P. Bhalerao, D. Blaudez, W. Boerjan, A. Brun, A. Brunner, V. Busov, M. Campbell, J. Carlson, M. Chalot, J. Chapman, G.-L. Chen, D. Cooper, P. M. Coutinho, J. Couturier, S. Covert, Q. Cronk, R. Cunningham, J. Davis, S. Degroeve, A. Déjardin, C. dePamphilis, J. Detter, B. Dirks, I. Dubchak, S. Duplessis, J. Ehlting, B. Ellis, K. Gendler, D. Goodstein, M. Gribskov, J. Grimwood, A. Groover, L. Gunter, B. Hamberger, B. Heinze, Y. Helariutta, B. Henrissat, D. Holligan, R. Holt, W. Huang, N. Islam-Faridi, S. Jones, M. Jones-Rhoades, R. Jorgensen, C. Joshi, J. Kangasjärvi, J. Karlsson, C. Kelleher, R. Kirkpatrick, M. Kirst, A. Kohler, U. Kalluri, F. Larimer, J. Leebens-Mack, J.-C. Leplé, P. Locascio, Y. Lou, S. Lucas, F. Martin, B. Montanini, C. Napoli, D. R. Nelson, C. Nelson, 
K. Nieminen, O. Nilsson, V. Pereda, G. Peter, R. Philippe, G. Pilate, A. Poliakov, J. Razumovskaya, P. Richardson, C. Rinaldi, K. Ritland, P. Rouzé, D. Ryaboy, J. Schmutz, J. Schrader, B. Segerman, H. Shin, A. Siddiqui, F. Sterky, A. Terry, C.-J. Tsai, E. Uberbacher, P. Unneberg, J. Vahala, K. Wall, S. Wessler, G. Yang, T. Yin, C. Douglas, M. Marra, G. Sandberg, Y. Van de Peer, and D. Rokhsar. 2006. 'The Genome of Black Cottonwood, Populus trichocarpa (Torr. \&amp; Gray)', Science, 313: 1596604.

Van der Knaap, E., Kim, J. H., and Kende, H. 2000. 'A Novel Gibberellin-Induced Gene from Rice and Its Potential Regulatory Role in Stem Growth', Plant Physiology, 122: 695-704.

Wang, F., Qiu, N., Ding, Q., Li, J., Zhang, Y., Li, H., and Gao J. 2014. 'Genomewide identification and analysis of the growth-regulating factor family in Chinese cabbage (Brassica rapa L. ssp. pekinensis)', Biomed Central Genomics, 15: 807-19.

Wang, Y., Wang, Z., Yang, W., Xie, X., Cheng, H., Qin, Li., Tang, G., and Huang, B. 2019. 'Degradation of Fungal MicroRNAs Triggered by Short Tandem Target Mimics Is via the Small-RNA-Degrading Nuclease', Applied Environmental Microbiology, 85: 1-13.

Yan, J., Gu, Y., Jia, X., Kang, W., Pan, S., Tang, X., Chen, X., and Tang, G. 2012. 'Effective Small RNA Destruction by the Expression of a Short Tandem Target Mimic in Arabidopsis', The Plant Cell, 24: 415-27.

Zhang, D., Sun, W., Singh, R., Zheng, Y., Cao, Z., Li, M., Lunde, C., Hake, S., and Zhang. Z. 2018. 'GRF-interacting factor1 Regulates Shoot Architecture and Meristem Determinacy in Maize', The Plant Cell, 30: 360-74. 